\title{
Unstable normalized standing waves for the space periodic NLS
}

\author{
Nils Ackermann* Tobias Weth
}

\begin{abstract}
For the stationary nonlinear Schrödinger equation $-\Delta u+V(x) u-f(u)=$ $\lambda u$ with periodic potential $V$ we study the existence and stability properties of multibump solutions with prescribed $L^{2}$-norm. To this end we introduce a new nondegeneracy condition and develop new superposition techniques which allow to match the $L^{2}$-constraint. In this way we obtain the existence of infinitely many geometrically distinct solutions to the stationary problem. We then calculate the Morse index of these solutions with respect to the restriction of the underlying energy functional to the associated $L^{2}$-sphere, and we show their orbital instability with respect to the Schrödinger flow. Our results apply in both, the mass-subcritical and the mass-supercritical regime.

Keywords: Nonlinear Schrödinger equation; periodic potential; standing wave solution; orbitally unstable solution; multibump construction; prescribed norm
\end{abstract}

MSC: 35J91, 35Q55; 35J20

\section{Introduction}

Suppose that $N \in \mathbb{N}$ and consider the stationary nonlinear Schrödinger equation with prescribed $L^{2}$-norm

$\left(P_{\alpha}\right) \quad-\Delta u+V(x) u-f(u)=\lambda u, \quad u \in H^{1}\left(\mathbb{R}^{N}\right), \quad|u|_{2}^{2}=\alpha$

which we will call the constrained equation. Here $|\cdot|_{2}$ denotes the standard $L^{2}$-norm, $V \in L^{\infty}\left(\mathbb{R}^{N}\right)$ is periodic in all coordinates, $f$ is a superlinear nonlinearity of class $C^{1}$ with Sobolev-subcritical growth, $\alpha>0$ is given, $u$ is the unknown weak solution and $\lambda \in \mathbb{R}$ is an unknown parameter. Solutions to Equation $\left(P_{\alpha}\right)$ are standing wave solutions

\footnotetext{
*Supported by CONACYT grant 237661, UNAM-DGAPA-PAPIIT grant IN100718 and the program UNAM-DGAPA-PASPA (Mexico)
} 
for the time-dependent Schrödinger Equation modeling a Bose-Einstein condensate in a periodic optical lattice $[4,7,15,18,20,28,37,41,43]$. In this model $\alpha$ is proportional to the total number of atoms in the condensate.

Set

$$
\Sigma_{\alpha}:=\left\{\left.u \in H^{1}\left(\mathbb{R}^{N}\right)|| u\right|_{2} ^{2}=\alpha\right\}
$$

for $\alpha>0$. Define the functional $\Phi: H^{1}\left(\mathbb{R}^{N}\right) \rightarrow \mathbb{R}$ by

$$
\Phi(u):=\frac{1}{2} \int_{\mathbb{R}^{N}}\left(|\nabla u|^{2}+V u^{2}\right)-\int_{\mathbb{R}^{N}} F(u),
$$

where we have set $F(s):=\int_{0}^{s} f$. Then the pair $(u, \lambda)$ is a weak solution of $\left(P_{\alpha}\right)$ if and only if $u$ is a critical point of the restriction of $\Phi$ to $\Sigma_{\alpha}$ with Lagrange multiplier $\lambda$.

Not assuming periodicity of $V$ but instead $\sup _{\mathbb{R}^{N}} V=\lim _{|x| \rightarrow \infty} V(x)$, the existence of a minimizer of $\Phi$ on $\Sigma_{\alpha}$ in the mass-subcritical case was shown under additional assumptions on the growth of the nonlinearity $f$ by Lions [36]; see also [33] for a different approach. For constant $V$ solutions of $\left(P_{\alpha}\right)$ are constructed in the mass-supercritical case in $[8,9,32]$; here the corresponding critical points of $\left.\Phi\right|_{\Sigma_{\alpha}}$ are not local minimizers. In $[11,12,21,22]$ local minimizers are found in the mass-supercritical case under spatially constraining potentials.

The structure of the solution set of the constrained equation is rather poorly understood up to now in the case where $V \in L^{\infty}\left(\mathbb{R}^{N}\right)$ is not constant, but 1-periodic in all coordinates. In contrast, a large amount of information is available for the free equation

$$
-\Delta u+V(x) u=f(u), \quad u \in H^{1}\left(\mathbb{R}^{N}\right),
$$

where essentially the parameter $\lambda$ is fixed but the $L^{2}$-norm is not prescribed anymore. Of particular interest for us are the results on the existence of so-called multibump solutions. In $[1,3,5,6,14,34,45,46,48]$, an infinite number of solutions are built using nonlinear superposition of translates of a special solution which satisfies a nondegeneracy condition of some form.

The main goal of the present work is to apply nonlinear superposition techniques to the constrained problem with periodic $V$ to obtain an infinity of $L^{2}$-normalized solutions in the form of multibump solutions. We succeed in doing this, but have to impose a stricter nondegeneracy condition than in the case of the free equation which nevertheless is fulfilled in many situations. This provides, as far as we know, the first result on multibump solutions for the constrained problem, and also the first multiplicity result in the case of a nonconstant periodic potential $V$. We also compute the Morse index of these normalized multibump solutions with respect to the restricted functional $\left.\Phi\right|_{\Sigma_{\alpha}}$, and we will use the Morse index information to derive orbital instability of the multibump solutions.

To state our results, we need the following hypotheses. We consider, as usual, the critical Sobolev exponent defined by $2^{*}:=\frac{2 N}{N-2}$ in case $N \geq 3$ and $2^{*}:=\infty$ in case $N=1,2$. 
(H1) $V \in L^{\infty}\left(\mathbb{R}^{N}\right)$

(H2) $V$ is 1-periodic in all coordinates;

(H3) $f \in C^{1}(\mathbb{R}), f(0)=f^{\prime}(0)=0$,

$$
\lim _{s \rightarrow \infty} \frac{f^{\prime}(s)}{|s|^{2^{*}-2}}=0
$$

if $N \geq 3$, and there is $p>2$ such that

$$
\lim _{s \rightarrow \infty} \frac{f^{\prime}(s)}{|s|^{p-2}}=0
$$

if $N=1$ or $N=2$.

Throughout this paper we assume (H1) and (H3). It is well known that $\Phi$ is well defined by (1.2) and of class $C^{2}$. The standard example for a function satisfying (H3) is $f(s):=|s|^{p-2} s$ with $p \in\left(2,2^{*}\right)$. In the following, we let $H^{-1}\left(\mathbb{R}^{N}\right)$ denote the topological dual of $H^{1}\left(\mathbb{R}^{N}\right)$. For our main result, we need the notion of a fully nondegenerate critical point of $\left.\Phi\right|_{\Sigma_{\alpha}}$.

Definition 1.1. Assume (H1) and (H3). For $\alpha>0$, a critical point $u \in H^{1}\left(\mathbb{R}^{N}\right)$ of $\left.\Phi\right|_{\Sigma_{\alpha}}$ with Lagrangian multiplier $\lambda$ will be called fully nondegenerate if for every $g \in H^{-1}\left(\mathbb{R}^{N}\right)$ there exists a unique weak solution $z_{g} \in H^{1}\left(\mathbb{R}^{N}\right)$ of the linearized equation

$$
-\Delta z_{g}+[V-\lambda] z_{g}-f^{\prime}(u) z_{g}=g \quad \text { in } \mathbb{R}^{N},
$$

and if in the case $g=u$ we have $\int_{\mathbb{R}^{N}} u z_{u} \neq 0$. Here, as usual, we regard $H^{1}\left(\mathbb{R}^{N}\right)$ as a subspace of $H^{-1}\left(\mathbb{R}^{N}\right)$, so $u \in H^{-1}\left(\mathbb{R}^{N}\right)$.

As we shall see in Section 2 below, the full nondegeneracy of a critical point $u \in$ $H^{1}\left(\mathbb{R}^{N}\right)$ of $\left.\Phi\right|_{\Sigma_{\alpha}}$ with Lagrangian multiplier $\lambda$ implies the nondegeneracy of the Hessian of $\left.\Phi\right|_{\Sigma_{\alpha}}$ at $u$. By definition, this Hessian is the bilinear form

$$
(v, w) \mapsto \int_{\mathbb{R}^{N}}\left(\nabla v \nabla w+[V-\lambda] v w-f^{\prime}(u) v w\right)
$$

defined on the tangent space

$$
T_{u} \Sigma_{\alpha}=\left\{v \in H^{1}\left(\mathbb{R}^{N}\right) \mid(v, u)_{2}=0\right\},
$$

see Definition 2.5 below. Here $(\cdot, \cdot)_{2}$ denotes the standard scalar product in $L^{2}\left(\mathbb{R}^{N}\right)$. We also need to fix the following elementary notation. If $n \in \mathbb{N}$ and $a=\left(a^{1}, a^{2}, \ldots, a^{n}\right) \in$ $\left(\mathbb{Z}^{N}\right)^{n}$ is a tuple of $n$ elements from $\mathbb{Z}^{N}$, denote

$$
d(a):=\min _{i \neq j}\left|a^{i}-a^{j}\right| .
$$

Moreover, for $b \in \mathbb{R}^{N}$ we denote by $\mathcal{T}_{b}$ the associated translation operator, i.e., for $u: \mathbb{R}^{N} \rightarrow \mathbb{R}$ the function $\mathcal{T}_{b} u: \mathbb{R}^{N} \rightarrow \mathbb{R}$ is given by

$$
\mathcal{T}_{b} u(x):=u(x-b), \quad \text { for } x \in \mathbb{R}^{N} .
$$

Our first main result is the following. 
Theorem 1.2 (Multibump Solutions). Assume (H1)-(H3) and fix $\alpha>0, n \in \mathbb{N}, n \geq 2$. Moreover, suppose that $\bar{u}$ is a fully nondegenerate critical point of $\left.\Phi\right|_{\Sigma_{\alpha / n}}$ with Lagrangian multiplier $\bar{\lambda}$. Then for every $\varepsilon>0$ there exists $R_{\varepsilon}>0$ such that for every $a \in\left(\mathbb{Z}^{N}\right)^{n}$ with $d(a) \geq R_{\varepsilon}$ there is a critical point $u_{a}$ of $\left.\Phi\right|_{\Sigma_{\alpha}}$ with Lagrange multiplier $\lambda_{a}$ such that

$$
\left\|u_{a}-\sum_{i=1}^{n} \mathcal{T}_{a^{i}} \bar{u}\right\|_{H^{1}\left(\mathbb{R}^{N}\right)} \leq \varepsilon \quad \text { and } \quad\left|\lambda_{a}-\bar{\lambda}\right| \leq \varepsilon .
$$

If $\varepsilon$ is chosen small enough then $u_{a}$ is unique. Moreover, if $\bar{u}$ is a positive function and $f(\bar{u}) \geq 0$ on $\mathbb{R}^{N}, f(\bar{u}) \not \equiv 0$, then $u_{a}$ is positive as well.

The proof of Theorem 1.2 is based on a general Shadowing Lemma, a simple consequence of Banach's Fixed Point Theorem, applied to approximate zeros of the gradient of the extended Lagrangian $G_{\alpha}$ for the constrained variational problem on $\Sigma_{\alpha}$. If $\bar{u}$ is a nondegenerate local minimum of $\Phi$ on $\Sigma_{\alpha / n}$ then it is easy to see that the sum $\tilde{u}$ of $n$ translates of $\bar{u}$ is an approximate zero of $\nabla G_{\alpha}$ if these translates are far enough apart from each other. The Shadowing Lemma implies that to obtain a zero of $\nabla G_{\alpha}$ near $\tilde{u}$ it is sufficient to prove that $\mathrm{D}^{2} G_{\alpha}(\tilde{u})$ is invertible and that the norm of its inverse is bounded appropriately. This step is the main difficulty and requires the assumption of full nondegeneracy of $\bar{u}$.

Our next result is concerned with the Morse index of the solutions $u_{a}$ given in Theorem 1.2 with respect to the functional $\left.\Phi\right|_{\Sigma_{\alpha}}$. For this we recall that the Morse index $m(u)$ of a critical point $u$ of $\left.\Phi\right|_{\Sigma_{\alpha}}$ with Lagrangian multiplier $\lambda$ is defined as the maximal dimension of a subspace $W \subset T_{u} \Sigma_{\alpha}$ such that the quadratic form in (1.4) is negative definite on $W$. If such a maximal dimension does not exist, one sets $m(u)=\infty$. We also introduce the following additional assumption.

(H4) $f(s) /|s|$ is nondecreasing in $\mathbb{R}$ and $f(s) s>0$ for all $s \neq 0$.

Theorem 1.3. Assume (H1)-(H3), fix $\alpha>0, n \in \mathbb{N}, n \geq 2$, and suppose that $\bar{u}$ is a fully nondegenerate critical point of $\left.\Phi\right|_{\Sigma_{\alpha / n}}$ with Lagrangian multiplier $\bar{\lambda}$ and finite Morse index $m(\bar{u})$. Moreover, let $z_{\bar{u}}$ be given as in Definition 1.1 with $u=\bar{u}$. Then the critical points $u_{a}$ found in Theorem 1.2 have, for small $\varepsilon$, the following Morse index $m\left(u_{a}\right)$ with respect to $\left.\Phi\right|_{\Sigma_{\alpha}}$ :

$$
m\left(u_{a}\right)= \begin{cases}n(m(\bar{u})+1)-1 & \text { if }\left(\bar{u}, z_{\bar{u}}\right)_{2}<0 \\ n m(\bar{u}) & \text { if }\left(\bar{u}, z_{\bar{u}}\right)_{2}>0 .\end{cases}
$$

If moreover (H4) holds true, then $m\left(u_{a}\right)>0$.

The key rôle of the sign of the scalar product $\left(\bar{u}, z_{\bar{u}}\right)_{2}$ in this theorem is not surprising since it is closely related to variational properties of the underlying critical point $\bar{u}$. More precisely, we shall see in Lemma 2.6 below that it determines the relationship between the Morse index of $\bar{u}$ with respect to $\left.\Phi\right|_{\Sigma_{\alpha / n}}$ and its free Morse index with respect to the functional $u \mapsto \Phi(u)-\bar{\lambda}|u|_{2}^{2}$ on $H^{1}\left(\mathbb{R}^{N}\right)$. 
We now consider the special case where (H4) holds true and $\bar{u}$ is a nondegenerate local minimum of $\left.\Phi\right|_{\Sigma_{\alpha / n}}$. By a nondegenerate local minimum we mean a critical point $\bar{u}$ of $\left.\Phi\right|_{\Sigma_{\alpha / n}}$ with Lagrangian multiplier $\bar{\lambda}$ such that the quadratic form in (1.4) is positive definite on $T_{u} \Sigma_{\alpha / n}$. In this case, we shall see in Section 2 below that $\bar{u}$ is fully nondegenerate, and we will deduce the following corollary from Theorems 1.2 and 1.3 in Section 4.

Corollary 1.4. Assume (H1)-(H4) and fix $\alpha>0, n \in \mathbb{N}, n \geq 2$. Moreover, suppose that $\bar{u}$ is a nondegenerate local minimum of $\left.\Phi\right|_{\Sigma_{\alpha / n}}$ with Lagrangian multiplier $\bar{\lambda}$. Then for every $\varepsilon>0$ there exists $R_{\varepsilon}>0$ such that for every $a \in\left(\mathbb{Z}^{N}\right)^{n}$ with $d(a) \geq R_{\varepsilon}$ there is a critical point $u_{a}$ of $\left.\Phi\right|_{\Sigma_{\alpha}}$ with Lagrange multiplier $\lambda_{a}$ such that

$$
\left\|u_{a}-\sum_{i=1}^{n} \mathcal{T}_{a^{i}} \bar{u}\right\|_{H^{1}\left(\mathbb{R}^{N}\right)} \leq \varepsilon \quad \text { and } \quad\left|\lambda_{a}-\bar{\lambda}\right| \leq \varepsilon .
$$

If $\varepsilon$ is chosen small enough then $u_{a}$ is unique. Moreover, $u_{a}$ does not change sign and has Morse index $m\left(u_{a}\right)=n-1$ with respect to $\left.\Phi\right|_{\Sigma_{\alpha}}$.

Next we present an example where the nondegeneracy hypotheses of the previous theorems can be verified. For this we make the following assumptions.

(H5) $V \in C^{2}\left(\mathbb{R}^{N}\right)$ is 1-periodic in all coordinates, positive, and has a nondegenerate critical point at some point $x_{0} \in \mathbb{R}^{N}$.

(H6) $f(s)=|s|^{p-2} s$ for some $p \in\left(2,2^{*}\right) \backslash\left\{2+\frac{4}{N}\right\}$.

We then consider the constrained singularly perturbed equation

$$
\left(P_{\alpha, \varepsilon}\right) \quad-\varepsilon^{2} \Delta u+V(x) u-|u|^{p-2} u=\lambda u, \quad u \in H^{1}\left(\mathbb{R}^{N}\right), \quad|u|_{2}^{2}=\alpha
$$

in the semiclassical limit $\varepsilon \rightarrow 0$. Its weak solutions correspond, for each $\varepsilon>0$, to critical points and Lagrange multipliers of the restriction of the functional

$$
\Phi_{\varepsilon}: H^{1}\left(\mathbb{R}^{N}\right) \rightarrow \mathbb{R}, \quad \Phi_{\varepsilon}(u):=\frac{1}{2} \int_{\mathbb{R}^{N}}\left(\varepsilon^{2}|\nabla u|^{2}+V u^{2}\right)-\frac{1}{p} \int_{\mathbb{R}^{N}}|u|^{p}
$$

to $\Sigma_{\alpha}$. We also consider the related free problem

$$
-\varepsilon^{2} \Delta u+V(x) u=|u|^{p-2} u, \quad u \in H^{1}\left(\mathbb{R}^{N}\right),
$$

whose weak solutions coincide with critical points of $\Phi_{\varepsilon}$, for every $\varepsilon>0$. It is well known (see [26]) that there exists a locally unique curve of solutions of $\left(F_{\varepsilon}\right)$ that concentrate near $x_{0}$ as $\varepsilon \rightarrow 0$. For our purposes we need to show additional properties of these solutions.

Theorem 1.5. Assume (H5) and (H6). Then there exist $\varepsilon_{0}>0$ and a continuous map $\left(0, \varepsilon_{0}\right) \rightarrow H^{1}\left(\mathbb{R}^{N}\right), \varepsilon \rightarrow \bar{u}_{\varepsilon}$, such that the following properties hold true: 
(i) for each $\varepsilon \in\left(0, \varepsilon_{0}\right)$ the function $\bar{u}_{\varepsilon}$ is a positive solution of $\left(F_{\varepsilon}\right)$;

(ii) as $\varepsilon \rightarrow 0$, the functions $x \mapsto \bar{u}_{\varepsilon}$ concentrates near $x_{0}$ in the sense that the functions $x \mapsto \bar{u}_{\varepsilon}\left(x_{0}+\varepsilon x\right)$ converge in $H^{1}\left(\mathbb{R}^{N}\right)$ to the unique radial positive solution $u_{0} \in$ $H^{1}\left(\mathbb{R}^{N}\right)$ of the equation $-\Delta u_{0}+V\left(x_{0}\right) u_{0}=u_{0}^{p-1}$ in $\mathbb{R}^{N}$;

(iii) $\left|\bar{u}_{\varepsilon}\right|_{2}^{2} \rightarrow 0$ as $\varepsilon \rightarrow 0$;

(iv) for each $\varepsilon \in\left(0, \varepsilon_{0}\right)$ the function $\bar{u}_{\varepsilon}$ is a fully nondegenerate critical point of the restriction of $\Phi_{\varepsilon}$ to $\Sigma_{\left|\bar{u}_{\varepsilon}\right|_{2}^{2}}$ with Morse index

$$
m\left(\bar{u}_{\varepsilon}\right)= \begin{cases}m_{V} & \text { if } 2<p<2+\frac{4}{N}, \\ m_{V}+1 & \text { if } 2+\frac{4}{N}<p<2^{*} .\end{cases}
$$

Here $m_{V}$ denotes the number of negative eigenvalues of the Hessian of $V$ at $x_{0}$.

We emphasize that properties (i)-(ii) were already proved in [26], and that (iii) follows from (ii) by a simple change of variable. For our purposes, the property (iv) is of key importance. We shall also see in Section 5 below that, for $\varepsilon \in\left(0, \varepsilon_{0}\right)$,

$$
\left(\bar{u}_{\varepsilon}, z_{\bar{u}_{\varepsilon}}\right)_{2}<0 \text { if } 2<p<2+\frac{4}{N} \text { and }\left(\bar{u}_{\varepsilon}, z_{\bar{u}_{\varepsilon}}\right)_{2}>0 \text { if } 2+\frac{4}{N}<p<2^{*},
$$

where $z_{\bar{u}_{\varepsilon}}$ is given as in Definition 1.1 corresponding to $u=\bar{u}_{\varepsilon}$. Since the solutions $\bar{u}_{\varepsilon}$ in Theorem 1.5 depend continuously on $\varepsilon$ and $\left|\bar{u}_{\varepsilon}\right|_{2}^{2} \rightarrow 0$ as $\varepsilon \rightarrow 0$, we can find, for every $\alpha>0$ and large enough $n \in \mathbb{N}$, a number $\varepsilon_{n} \in\left(0, \varepsilon_{0}\right)$ such that $\left|\bar{u}_{\varepsilon_{n}}\right|_{2}^{2}=\alpha / n$. The combination of Theorems 1.2, 1.3, and 1.5 with (1.6) therefore yields the following corollary.

Corollary 1.6. Assume (H5) and (H6). Then for every $\alpha>0$ there exist $n_{\alpha} \in \mathbb{N}$ and a sequence $\varepsilon_{n} \rightarrow 0$ such that for every $n \geq n_{\alpha}$ the problem $\left(P_{\alpha, \varepsilon_{n}}\right)$ has infinitely many geometrically distinct positive solutions. More precisely, for every $n \in \mathbb{N}$ with $n \geq n_{\alpha}$, and every $\delta>0$ there exists $R_{\delta, n}>0$ such that for every $a \in\left(\mathbb{Z}^{N}\right)^{n}$ with $d(a) \geq R_{\delta, n}$ there is a critical point $u_{a}$ of $\left.\Phi_{\varepsilon_{n}}\right|_{\Sigma_{\alpha}}$ with Lagrange multiplier $\lambda_{a}$ such that

$$
\left\|u_{a}-\sum_{i=1}^{n} \mathcal{T}_{a^{i}} \bar{u}_{\varepsilon_{n}}\right\|_{H^{1}\left(\mathbb{R}^{N}\right)} \leq \delta \quad \text { and } \quad\left|\lambda_{a}\right| \leq \delta .
$$

If $\delta$ is chosen small enough then $u_{a}$ is unique. Moreover, $u_{a}$ is a positive function, and its Morse index with respect to $\left.\Phi\right|_{\Sigma_{\alpha}}$ is given by

$$
m\left(u_{a}\right)= \begin{cases}n\left(m_{V}+1\right)-1 & \text { if } 2<p<2+\frac{4}{N}, \\ n\left(m_{V}+1\right) & \text { if } 2+\frac{4}{N}<p<2^{*},\end{cases}
$$

where $m_{V}$ denotes the number of negative eigenvalues of the Hessian of $V$ at $x_{0}$. 
Our next result is concerned with the orbital instability of the normalized multibump solutions we have constructed in the previous theorems. For this we focus on odd nonlinearities $f$ in $\left(P_{\alpha}\right)$ satisfying (H3) and therefore assume

(H7) the function $f$ is odd.

We also assume (H1) and (H3), so $\Phi$ in (1.2) is a well defined $C^{2}$-functional. If $\varphi \in \Sigma_{\alpha}$ is a critical point of $\left.\Phi\right|_{\Sigma_{\alpha}}$ with Lagrangian multiplier $\lambda$, then the function

$$
u_{\varphi}: \mathbb{R} \times \mathbb{R}^{N} \rightarrow \mathbb{C}, \quad u_{\varphi}(t, x)=\varphi(x) \mathrm{e}^{i \lambda t}
$$

is a solution of the time-dependent nonlinear Schrödinger equation

$$
-i u_{t}=-\Delta u+V(x) u-g\left(|u|^{2}\right) u,
$$

where $g$ is defined by $f(t)=g\left(|t|^{2}\right) t$. Solutions of this special type are usually called solitary wave solutions. The solution $u_{\varphi}$ is called orbitally stable if for every $\varepsilon>0$ there exists $\delta>0$ such that every solution $u:\left[0, t_{0}\right) \rightarrow H^{1}\left(\mathbb{R}^{N}, \mathbb{C}\right)$ of $(1.8)$ with $\| u(0, \cdot)-$ $\varphi \|_{H^{1}}<\delta$ can be extended to a solution $[0, \infty) \rightarrow H^{1}\left(\mathbb{R}^{N}, \mathbb{C}\right)$ which satisfies

$$
\sup _{0<t<\infty} \inf _{s \in \mathbb{R}}\left\|u(t, \cdot)-u_{\varphi}(s, \cdot)\right\|_{H^{1}}<\varepsilon
$$

Otherwise, $u_{\varphi}$ is called orbitally unstable. We then have the following result.

Theorem 1.7. Assume (H1), (H3), and (H7), and suppose that $\varphi \in \Sigma_{\alpha}$ is a positive function which is a critical point of $\left.\Phi\right|_{\Sigma_{\alpha}}$ with positive Morse index and Lagrangian multiplier $\lambda<\inf \sigma_{\mathrm{ess}}(-\Delta+V)$. Then the corresponding solitary wave solution $u_{\varphi}$ of (1.8) is orbitally unstable.

Here and in the following, $\sigma_{\text {ess }}(-\Delta+V)$ denotes the essential spectrum of the Schrödinger operator $-\Delta+V$. We note that Theorem 1.7 neither requires periodicity of $V$, nor does it require the assumption on the oddness of a certain difference of numbers of eigenvalues in the seminal instability result in $[25$, p. 309]. Theorem 1.7 applies to the normalized multibump solutions constructed in Theorem 1.2 and Corollaries 1.4 and 1.6 in the case where the nonlinearity satisfies (H4) and (H7). In these cases, the extra assumption $\lambda<\inf \sigma_{\text {ess }}(-\Delta+V)$ follows from Lemma 2.9 below and the fact that the Lagrangian multipliers of the multibump solutions are arbitrarily close to the multiplier of the initial solution.

There are many results on the orbital stability and instability of the standing waves generated by solutions to $\left(P_{\alpha}\right)$, see $[13,24,28,31,50]$. However, none of these results covers the situation addressed in Theorem 1.7.

The paper is organized as follows. In Section 2 we collect some preliminary notions and observations. In particular, here we explain our new notions of fully nondegenerate restricted critical point and of the free Morse index. In Section 3 we then prove Theorem 1.2. In Section 4 we derive a general result on the Morse index of normalized multibump solutions which gives rise to Theorem 1.3. At the end of this section, we also 
complete the proof of Corollary 1.4. In Section 5, we analyze the singular perturbed problem $\left(F_{\varepsilon}\right)$ and we prove Theorem 1.5. In Section 6, we then prove the orbital instability result given in Theorem 1.7. Finally, in the Appendix we provide a computation of the free Morse index of the solutions $u_{\varepsilon}$ considered in Theorem 1.5. This computation is partly contained in [35, Proof of Theorem 2.5], but some details have been omitted there. We therefore provide a somewhat different argument in detail for the convenience of the reader.

We finally remark that the main results of our paper can be extended to more general nonlinearities. In particular, Theorem 1.2 has an abstract proof that extends to nonlinearities that also depend on $x, 1$-periodically in every coordinate. This proof also extends to nonlocal nonlinearities with convolution terms as in [31]. This follows from Brézis-Lieb type splitting properties for these nonlinearities that were proved in [1].

\subsection{Notation}

In the remainder of the paper, we write $|\cdot|_{p}$ for the standard $L^{p}\left(\mathbb{R}^{N}\right)$-norm, $1 \leq p \leq \infty$. We also use the notation $(\cdot, \cdot)_{2}$ for the standard $L^{2}\left(\mathbb{R}^{N}\right)$-scalar product. For the sake of brevity, we write $L^{2}$ in place of $L^{2}\left(\mathbb{R}^{N}\right)$ and $H^{k}$ in place of $H^{k}\left(\mathbb{R}^{N}\right)$, for $k \in \mathbb{N}$. By (H1), $-\Delta+V$ is a self adjoint operator in $L^{2}$ with domain $H^{2}$. Since we assume (H1) throughout the paper and $\lambda$ is a free parameter in $\left(P_{\alpha}\right)$, we may assume without loss of generality that $\gamma:=\min \sigma(-\Delta+V)>0$, where $\sigma(-\Delta+V)$ stands for the spectrum of $-\Delta+V$. Then $H^{1}$ is the form domain (the energy space) of $-\Delta+V$, and we may endow $H^{1}$ with the scalar product

$$
\langle u, v\rangle=\int_{\mathbb{R}^{N}}(\nabla u \cdot \nabla v+V u v), \quad u, v \in H^{1} .
$$

The norm $\|\cdot\|$ induced by $\langle\cdot, \cdot\rangle$ is equivalent to the standard norm on $H^{1}$. It will be convenient to denote $S:=(-\Delta+V)^{-1}$; then we have

$$
\langle u, v\rangle=\left(S^{-1 / 2} u, S^{-1 / 2} v\right)_{2} \quad \text { for } u, v \in H^{1} .
$$

We point out that, for a subspace $Z \subset H^{1}$, the notation $Z^{\perp}$ always refers to the orthogonal complement of $Z$ in $H^{1}$ with respect to the scalar product $\langle\cdot, \cdot\rangle$.

We recall that the spectrum $\sigma(-\Delta+V)$ is purely essential if (H2) is assumed. In this case, it also follows that all powers of $S$ are equivariant with respect to the action of $\mathbb{Z}^{N}$. Hence

$$
\left\langle\mathcal{T}_{a} v, \mathcal{T}_{a} w\right\rangle=\langle v, w\rangle \quad \forall v, w \in H^{1}, \forall a \in \mathbb{Z}^{N} .
$$

For any two normed spaces $X, Y$ the space of bounded linear operators from $X$ in $Y$ is denoted by $\mathcal{L}(X, Y)$, and we write $\mathcal{L}(X):=\mathcal{L}(X, X)$.

For a $C^{1}$-functional $\Theta$ defined on $H^{1}$, we let $\mathrm{d} \Theta: H^{1} \rightarrow\left(H^{1}\right)^{*}$ denote the derivative of $\Theta$ and $\nabla \Theta: H^{1} \rightarrow H^{1}$ the gradient with respect to the scalar product $\langle\cdot, \cdot\rangle$ defined in (1.9). Moreover, if $\Theta$ is of class $C^{2}$, then $\mathrm{d}^{2} \Theta(u): H^{1} \times H^{1} \rightarrow \mathbb{R}$ denotes the Hessian of $\Theta$ at a point $u \in H^{1}$, whereas $\mathrm{D}^{2} \Theta(u) \in \mathcal{L}\left(H^{1}\right)$ stands for the derivative of the gradient of $\Theta$ at $u$. We then have

$$
\left\langle\mathrm{D}^{2} \Theta(u) v, w\right\rangle=\mathrm{d}^{2} \Theta(u)[v, w] \quad \text { for } v, w \in H^{1} .
$$


Acknowledgement: The authors wish to thank the referee for his/her valuable comments and corrections.

\section{Some preliminary abstract results and notions}

In this section we state some abstract results which will be used in Section 3 in the proof of Theorem 1.2. We start with a standard corollary of Banach's fixed point theorem, which is sometimes referred to as a Shadowing Lemma.

Lemma 2.1. Let $(E,\|\cdot\|)$ be a Banach space, let $h: E \rightarrow E$ be continuously differentiable with derivative $\mathrm{d} h: E \rightarrow \mathcal{L}(E)$, and let $v_{0} \in E, \delta>0, q \in(0,1)$ satisfy the following:

(i) $T:=\mathrm{d} h\left(v_{0}\right) \in \mathcal{L}(E)$ is an isomorphism.

(ii) $\left\|h\left(v_{0}\right)\right\|<\frac{\delta(1-q)}{\left\|T^{-1}\right\|_{\mathcal{L}(E)}}$.

(iii) $\|\mathrm{d} h(y)-T\|_{\mathcal{L}(E)} \leq \frac{q}{\left\|T^{-1}\right\|_{\mathcal{L}(E)}}$ for $y \in B_{\delta}\left(v_{0}\right)$.

Then $h$ has a unique zero in $B_{\delta}\left(v_{0}\right)$.

The proof of this lemma is standard by showing that the map $y \mapsto y-T^{-1} h(y)$ defines a $q$-contraction on $\overline{B_{\delta}\left(v_{0}\right)}$. Applying Banach's fixed point theorem to this map gives rise to a unique zero of $h$ in $\overline{B_{\delta}\left(v_{0}\right)}$, and it easily follows from the above assumptions that this zero is contained in $B_{\delta}\left(v_{0}\right)$.

We will use the following immediate corollary of Lemma 2.1.

Corollary 2.2. Let $(E,\|\cdot\|)$ be a Banach space, let $h: E \rightarrow E$ be differentiable and such that its derivative $\mathrm{d} h: E \rightarrow \mathcal{L}(E)$ is uniformly continuous on bounded subsets of E. Moreover, let $\left(v_{k}\right)_{k}$ be a bounded sequence in $E$ such that

(i) $h\left(v_{k}\right) \rightarrow 0$ as $k \rightarrow \infty$;

(ii) $\mathrm{d} h\left(v_{k}\right) \in \mathcal{L}(E)$ is an isomorphism for $k \in \mathbb{N}$, and $\sup _{k \in \mathbb{N}}\left\|\mathrm{d} h\left(v_{k}\right)^{-1}\right\|_{\mathcal{L}(E)}<\infty$.

Then there exist $k_{0} \in \mathbb{N}$ and $u_{k} \in E, k \geq k_{0}$, with

$$
h\left(u_{k}\right)=0 \quad \text { for } k \geq k_{0}
$$

and

$$
\left\|u_{k}-v_{k}\right\| \rightarrow 0 \quad \text { as } k \rightarrow \infty .
$$

Moreover, the sequence $\left(u_{k}\right)_{k}$ is uniquely determined by properties (2.1), (2.2) for large $k$. 
In the remainder of this section, we collect some preliminary results and notions related to the functional $\Phi$ defined in (1.2) and its restrictions to spheres with respect to the $L^{2}\left(\mathbb{R}^{N}\right)$-norm. Recall that we are assuming conditions (H1) and (H3). We denote

$$
\Psi(u):=\int_{\mathbb{R}^{N}} F(u),
$$

SO

$$
\Phi(u)=\frac{1}{2}\|u\|^{2}-\Psi(u) .
$$

Following [1] we say that a map $g: X \rightarrow Y$ of Banach spaces $X$ and $Y$ BL-splits if $g\left(x_{n}\right)-g\left(x_{n}-x^{*}\right) \rightarrow g\left(x^{*}\right)$ in $Y$ if $x_{n} \rightarrow x^{*}$ in $X$. For example, by [1, Remark 3.3] the maps $\|\cdot\|^{2}$ and $|\cdot|_{2}^{2}$ BL-split. The next result about BL-splitting maps is less obvious:

Lemma 2.3. $\Psi, \nabla \Psi$ and $\mathrm{D}^{2} \Psi$ BL-split, and these maps are uniformly continuous on bounded subsets of $H^{1}$.

Before we give the proof we fix some $p \in\left(2,2^{*}\right)$ if $N \geq 3$ and we use $p$ given in (H3) if $N=1,2$. Using (H3) it is easy to construct, for every $\varepsilon>0$, functions $f_{i, \varepsilon} \in C^{1}(\mathbb{R})$, $i=1,2,3$, and a constant $C_{\varepsilon}>0$ such that

$$
f=\sum_{i=1}^{3} f_{i, \varepsilon}
$$

and such that

$$
\left|f_{1, \varepsilon}^{\prime}(s)\right| \leq \varepsilon, \quad\left|f_{2, \varepsilon}^{\prime}(s)\right| \leq C_{\varepsilon}|s|^{p-2}, \quad \text { and } \quad\left|f_{3, \varepsilon}^{\prime}(s)\right| \leq \varepsilon|s|^{2^{*}-2}, \quad \text { for all } s \in \mathbb{R} .
$$

If $N=1,2$ we simply choose $f_{3, \varepsilon} \equiv 0$ and ignore all terms that contain $2^{*}$.

Proof of Lemma 2.3. We only prove this in the case $N \geq 3$; the other cases are treated similarly. Consider $\left(u_{n}\right) \subseteq H^{1}$ such that $u_{n} \rightarrow u$. Then $\left(u_{n}\right)$ is bounded in $H^{1}$ and therefore also in $L^{q}$ for $q \in\left[2,2^{*}\right.$. For fixed $\varepsilon>0$ we have

$$
\left|f_{2, \varepsilon}^{\prime}\left(u_{n}\right)-f_{2, \varepsilon}^{\prime}\left(u_{n}-u\right)-f_{2, \varepsilon}^{\prime}(u)\right|_{p /(p-2)} \rightarrow 0
$$

by $[2$, Theorem 1.3$]$. On the other hand, there are varying constants $C>0$, independent of $\varepsilon$, such that

$$
\left|f_{1, \varepsilon}^{\prime}\left(u_{n}\right)-f_{1, \varepsilon}^{\prime}\left(u_{n}-u\right)-f_{1, \varepsilon}^{\prime}(u)\right|_{\infty} \leq C \varepsilon
$$

and

$$
\left|f_{3, \varepsilon}^{\prime}\left(u_{n}\right)-f_{3, \varepsilon}^{\prime}\left(u_{n}-u\right)-f_{3, \varepsilon}^{\prime}(u)\right|_{2^{*} /\left(2^{*}-2\right)} \leq C \varepsilon
$$

for all $n$. For all $v, w \in H^{1}$ with $\|v\|=\|w\|=1$ it follows that

$$
\begin{aligned}
& \left|\left\langle\left(\mathrm{D}^{2} \Psi\left(u_{n}\right)-\mathrm{D}^{2} \Psi\left(u_{n}-u\right)-\mathrm{D}^{2} \Psi(u)\right) v, w\right\rangle\right| \\
& \leq C \varepsilon|v|_{2}|w|_{2}+\left|f_{2, \varepsilon}^{\prime}\left(u_{n}\right)-f_{2, \varepsilon}^{\prime}\left(u_{n}-u\right)-f_{2, \varepsilon}^{\prime}(u)\right|_{p /(p-2)}|v|_{p}|w|_{p}+C \varepsilon|v|_{2^{*}}|w|_{2^{*}} \\
& \leq C(\varepsilon+o(1))
\end{aligned}
$$

and hence $\lim \sup _{n \rightarrow \infty}\left\|\mathrm{D}^{2} \Psi\left(u_{n}\right)-\mathrm{D}^{2} \Psi\left(u_{n}-u\right)-\mathrm{D}^{2} \Psi(u)\right\|_{\mathcal{L}\left(H^{1}\right)} \leq C \varepsilon$. Letting $\varepsilon \rightarrow 0$ we obtain the claim for $\mathrm{D}^{2} \Psi$. The proof for the uniform continuity of $\mathrm{D}^{2} \Psi$ on bounded subsets of $H^{1}$ is similar. Analogously, one treats the maps $\nabla \Psi$ and $\Psi$. 
We shall need the following simple consequence of assumption (H4).

Lemma 2.4. If conditions (H1) and (H3)-(H4) hold true and $u \in H^{1} \backslash\{0\}$ satisfies $\nabla \Phi(u)=\lambda$ Su for some $\lambda \in \mathbb{R}$, then

$$
\left\langle\left(\mathrm{D}^{2} \Phi(u)-\lambda S\right) u, u\right\rangle<0 .
$$

Proof. By (H3) and (H4), the map $s \mapsto f^{\prime}(s) s^{2}-f(s) s$ is nonnegative in $\mathbb{R}$, and it is positive on a nonempty open subset of $(-\varepsilon, \varepsilon) \backslash\{0\}$ for every $\varepsilon>0$. Moreover, since $u \in H^{1}$ is a weak solution of

$$
-\Delta u+[V(x)-\lambda] u=f(u) \quad \text { in } \mathbb{R}^{N}
$$

by assumption, standard elliptic regularity shows that $u$ is continuous and that $u(x) \rightarrow 0$ as $|x| \rightarrow \infty$. Consequently, we have

$$
\begin{aligned}
\left\langle\mathrm{D}^{2} \Phi(u) u, u\right\rangle-\lambda\langle S u, u\rangle & =\left\langle\mathrm{D}^{2} \Phi(u) u, u\right\rangle-\langle\nabla \Phi(u), u\rangle \\
& =\langle\nabla \Psi(u), u\rangle-\left\langle\mathrm{D}^{2} \Psi(u) u, u\right\rangle=\int_{\mathbb{R}^{N}}\left(f(u) u-f^{\prime}(u) u^{2}\right)<0,
\end{aligned}
$$

as claimed.

As before, for $\alpha>0$, we consider the sphere $\Sigma_{\alpha} \subset H^{1}$ as defined in (1.1), and we let $J_{\alpha}: \Sigma_{\alpha} \rightarrow \mathbb{R}$ denote the restriction of $\Phi$ to $\Sigma_{\alpha}$. We note that, for $u \in \Sigma_{\alpha}$, the tangent space of $\Sigma_{\alpha}$ at $u$ is given by

$$
T_{u} \Sigma_{\alpha}=\left\{v \in H^{1} \mid(v, u)_{2}=0\right\}=\left\{v \in H^{1} \mid\langle v, S u\rangle=0\right\} \subset H^{1},
$$

where latter equality follows from (1.10). If $u$ is a critical point of $J_{\alpha}$, we have

$$
\nabla \Phi(u)=\lambda S u
$$

for some $\lambda \in \mathbb{R}$, the corresponding Lagrange multiplier. Moreover, the Hessian $\mathrm{d}^{2} J_{\alpha}(u)$ is a well-defined quadratic form on $T_{u} \Sigma_{\alpha}$ given by

$$
\mathrm{d}^{2} J_{\alpha}(u)[v, w]=\left\langle\mathrm{D}^{2} \Phi(u) v, w\right\rangle-\lambda\langle S v, w\rangle \quad \text { for } v, w \in T_{u} \Sigma_{\alpha} .
$$

For the general definition of the Hessian of $C^{2}$-functionals on Banach manifolds at critical points, see e.g. [44, p. 307]. To see (2.7), one may argue with local coordinates for $\Sigma_{\alpha}$ at $u$, as is done, e.g., in [17, Theorem 8.9] in the finite dimensional case. Alternatively, to prove (2.7) we may consider smooth vector fields $\tilde{v}, \tilde{w}$ on $\Sigma_{\alpha}$ with $\tilde{v}(u)=v, \tilde{w}(u)=w$, and we extend $\tilde{v}, \tilde{w}$ arbitrarily as smooth vector fields $\tilde{v}, \tilde{w}: H^{1} \rightarrow H^{1}$. Using (2.6), we then have

$$
\begin{aligned}
\mathrm{d}^{2} J_{\alpha}(u)[v, w] & =\partial_{\tilde{v}} \partial_{\tilde{w}} \Phi(u)=\left.\partial_{\tilde{v}}\right|_{u}\langle\nabla \Phi, w\rangle=\left\langle\mathrm{D}^{2} \Phi(u) v, w\right\rangle+\langle\nabla \Phi(u), \mathrm{d} \tilde{w}(u) v\rangle \\
& =\left\langle\mathrm{D}^{2} \Phi(u) v, w\right\rangle+\lambda(u, \mathrm{~d} \tilde{w}(u) v)_{2}=\left\langle\mathrm{D}^{2} \Phi(u) v, w\right\rangle-\lambda(v, w)_{2},
\end{aligned}
$$

where the last equality follows from the fact that the function $u_{*} \mapsto h\left(u_{*}\right):=\left(u_{*}, \tilde{w}\left(u_{*}\right)\right)_{2}$ vanishes on $\Sigma_{\alpha}$ and therefore $0=\partial_{\tilde{v}} h(u)=(v, w)_{2}+(u, \mathrm{~d} w(u) v)_{2}$.

We need the following definitions. 
Definition 2.5. Let $u \in H^{1}$ be a critical point of $J_{\alpha}$ with Lagrange multiplier $\lambda$. Put $\Lambda:=T_{u} \Sigma_{\alpha}$ and let $P \in \mathcal{L}\left(H^{1}, \Lambda\right)$ denote the $\langle\cdot, \cdot\rangle$-orthogonal projection onto $\Lambda$. Moreover, put $B:=\mathrm{D}^{2} \Phi(u)-\lambda S$.

(a) The Morse index $m(u) \in \mathbb{N} \cup\{0, \infty\}$ of $u$ with respect to $J_{\alpha}$ is defined as $m(u):=\sup \{\operatorname{dim} Z \mid Z$ subspace of $\Lambda$ with $\langle B v, v\rangle<0$ for all $v \in Z \backslash\{0\}\}$.

(b) The free Morse index $m_{\mathrm{f}}(u) \in \mathbb{N} \cup\{0, \infty\}$ of $u$ is defined as $m_{\mathrm{f}}(u):=\sup \left\{\operatorname{dim} Z \mid Z\right.$ subspace of $H^{1}$ with $\langle B v, v\rangle<0$ for all $\left.v \in Z \backslash\{0\}\right\}$.

(c) We call $u$ a nondegenerate critical point of $J_{\alpha}$ if $\left.P B\right|_{\Lambda}$ is an isomorphism of $\Lambda$.

(d) We call $u$ freely nondegenerate if $B$ is an isomorphism of $H^{1}$. In this case we put

$$
z_{u}:=B^{-1} S u \in H^{1} .
$$

For a critical point $u \in H^{1}$ of $J_{\alpha}$, it is clear that

$$
m_{\mathrm{f}}(u)=m(u) \quad \text { or } \quad m_{\mathrm{f}}(u)=m(u)+1 .
$$

In the case where $u$ is freely nondegenerate, the scalar product $\left(z_{u}, u\right)_{2}$ determines whether $u$ is nondegenerate and which case occurs in (2.8). More precisely, we have the following simple but important lemma.

Lemma 2.6. Let $u \in H^{1}$ be a freely nondegenerate critical point of $J_{\alpha}$ with Lagrange multiplier $\lambda$.

(a) $u$ is nondegenerate if and only if $\left(z_{u}, u\right)_{2} \neq 0$.

(b) If $m(u)$ is finite and $\left(z_{u}, u\right)_{2}>0$, then $m_{\mathrm{f}}(u)=m(u)$.

(c) If $m(u)$ is finite and $\left(z_{u}, u\right)_{2}<0$, then $m_{\mathrm{f}}(u)=m(u)+1$.

Proof. In the following, we let $\mathcal{N}(L)$ denote the kernel and $\mathcal{R}(L)$ denote the range of a linear operator $L$. Moreover, we let $B, P$ and $\Lambda$ be as in Definition 2.5.

(a): By definition, we have $z_{u}=B^{-1} S u \in \mathcal{N}(P B) \backslash\{0\}$. Moreover, we have $\operatorname{dim} \mathcal{N}(P B)=1$ since $B: H^{1} \rightarrow H^{1}$ is an isomorphism. Consequently,

$$
\mathcal{N}(P B)=\operatorname{span}\left(z_{u}\right) \quad \text { and } \quad \mathcal{R}(P B)=\Lambda .
$$

Now, again by definition, $u$ is nondegenerate if and only if $\left.P B\right|_{\Lambda}: \Lambda \rightarrow \Lambda$ is an isomorphism, and this holds true if and only if $H^{1}=\operatorname{span}\left(z_{u}\right) \oplus \Lambda$. By (2.5), the latter property is equivalent to $\left(z_{u}, u\right)_{2} \neq 0$.

(b) and (c): Since codim $\Lambda=1$ and $z_{u} \notin \Lambda$, there are, for every $\phi \in H^{1}$, unique elements $\mu \in \mathbb{R}$ and $w \in \Lambda$ such that

$$
\phi=\mu z_{u}+w
$$


Recall that $\operatorname{span}(S u)=\mathcal{N}(P)=\Lambda^{\perp}$. We therefore have the representation

$$
\begin{aligned}
\langle B \phi, \phi\rangle & =\mu^{2}\left\langle B z_{u}, z_{u}\right\rangle+2 \mu\left\langle B z_{u}, w\right\rangle+\langle B w, w\rangle \\
& =\mu^{2}\left\langle S u, z_{u}\right\rangle+2 \mu\langle S u, w\rangle+\langle B w, w\rangle \\
& =\mu^{2}\left(z_{u}, u\right)_{2}+\langle B w, w\rangle .
\end{aligned}
$$

To see (b), recall that the definition of $m(u)$ implies the existence of a subspace $Z \subset \Lambda$ of codimension $m(u)$ in $\Lambda$ such that $\langle B \phi, \phi\rangle \geq 0$ for all $\phi \in Z$. Since $z_{u} \notin \Lambda$, the space $\widetilde{Z}:=\operatorname{span}\left(z_{u}\right) \oplus Z$ has at most codimension $m(u)$ in $H^{1}$. Moreover, in the representation (2.9) for $\phi \in \widetilde{Z}$ we find $w \in Z$. Therefore, (2.10) yields $\langle B \phi, \phi\rangle \geq\langle B w, w\rangle \geq 0$. This implies $m_{\mathrm{f}}(u) \leq m(u)$, and thus equality follows by $(2.8)$.

To see (c), let $Z \subset \Lambda$ be an $m(u)$-dimensional subspace such that $\langle B w, w\rangle<0$ for all $w \in Z \backslash\{0\}$. Put $\widetilde{Z}:=\operatorname{span}\left(z_{u}\right) \oplus Z$. Then $\operatorname{dim} \widetilde{Z}=m(u)+1$, and for the representation (2.9) for $\phi \in \widetilde{Z} \backslash\{0\}$ we find $w \in Z$. Then (2.10) implies $\langle B \phi, \phi\rangle<0$ since either $\mu \neq 0$ or $w \in Z \backslash\{0\}$. Consequently, $m_{\mathrm{f}}(u) \geq m(u)+1$, and thus equality follows by (2.8).

Parts (b) and (c) of Lemma 2.6 can also be derived from [38, (2.7) of Theorem 2], see also [39]. For the convenience of the reader we gave a simple direct proof.

Definition 2.7. A critical point $u \in H^{1}$ of $J_{\alpha}$ will be called fully nondegenerate if $u$ is freely nondegenerate and the equivalent properties in Lemma 2.6(a) hold true.

Definition 2.7 is consistent with Definition 1.1, as the function $z_{u}=B^{-1} S u$ defined in Definition 2.5 is uniquely determined as the weak solution of (1.3) with $g=u$.

In the next lemma, we show that nondegenerate local minima of $J_{\alpha}$ are fully nondegenerate critical points.

Lemma 2.8. Suppose that (H4) holds true, and let $u \in H^{1}$ be a nondegenerate critical point of $J_{\alpha}$ with $m(u)=0$ (i.e., $u$ is a nondegenerate local minimum of $J_{\alpha}$ ). Then $u$ is fully nondegenerate, and either $u$ or $-u$ is a positive function.

Proof. We continue using the notation from the proof of Lemma 2.6. Since $u$ is nondegenerate, we have $\Lambda=\mathcal{R}\left(\left.P B\right|_{\Lambda}\right)$ and therefore $H^{1}=\mathcal{N}(P)+\mathcal{R}\left(\left.B\right|_{\Lambda}\right)$. This implies $\operatorname{codim} \mathcal{R}(B) \leq \operatorname{codim} \mathcal{R}\left(\left.B\right|_{\Lambda}\right) \leq 1$ and hence that $\mathcal{R}(B)$ is closed. Since $\left.P B\right|_{\Lambda}$ is injective, $\mathcal{N}(B) \cap \Lambda=\{0\}$ and hence $\operatorname{dim} \mathcal{N}(B) \leq 1$. If $\operatorname{dim} \mathcal{N}(B)=1$ were true, then we would have $H^{1}=\mathcal{N}(B) \oplus \Lambda$. Since the quadratic form $\langle B \cdot, \cdot\rangle$ is positive definite on $\Lambda$ it would be positive semidefinite on $H^{1}$, in contradiction with Lemma 2.4. Therefore $\mathcal{N}(B)=\{0\}$ and $B$, being symmetric with closed range, is an isomorphism. Hence $u$ is freely nondegenerate, and thus it is also fully nondegenerate.

Next, we suppose by contradiction that $u$ changes sign. A variant of the proof of Lemma 2.4 then shows that the quadratic form $\langle B \cdot, \cdot\rangle$ is negative definite on the twodimensional subspace $\operatorname{span}\left(u^{+}, u^{-}\right) \subset H^{1}$, where $u^{ \pm}:=\max \{0, \pm u\}$ denotes the positive, respectively negative part of $u$. Since this space has a nontrivial intersection with $\Lambda$, we thus obtain a contradiction to the assumption $m(u)=0$.

Next we add an observation for the case where $u$ is a fully nondegenerate critical point of $J_{\alpha}$ and a positive function. 
Lemma 2.9. Let $u \in H^{1}$ be a fully nondegenerate critical point of $J_{\alpha}$ with Lagrangian multiplier $\lambda$ such that $u$ is a positive function and $f(u) \geq 0$ on $\mathbb{R}^{N}, f(u) \not \equiv 0$. Then we have

$$
\lambda<\inf \sigma(-\Delta+V) .
$$

Proof. Since $u$ is freely nondegenerate, we see that

$$
\lambda \notin \sigma\left(-\Delta+V-f^{\prime}(u)\right) .
$$

Moreover, $u(x) \rightarrow 0$ as $|x| \rightarrow \infty$ by standard elliptic estimates, and the same is true for the functions $x \mapsto f^{\prime}(u(x)), x \mapsto \frac{f(u(x))}{u(x)}$. Consequently, by (2.12), Theorem 14.6 and the proof of Theorem 14.9 in [29] we have for $L_{0}:=-\Delta+V$ and $L:=-\Delta+V-\frac{f(u)}{u}$ that

$$
\lambda \notin \sigma_{\mathrm{ess}}\left(-\Delta+V-f^{\prime}(u)\right)=\sigma_{\mathrm{ess}}\left(L_{0}\right)=\sigma_{\mathrm{ess}}(L),
$$

where $\sigma_{\text {ess }}$ denotes the essential spectrum. Since $u$ is an eigenfunction of the Schrödinger operator $L$ corresponding to the eigenvalue $\lambda$, it follows that $\lambda$ is isolated in $\sigma(L)$. Since moreover $u$ is positive, it is then easy to see that $\lambda=\inf \sigma(L)$, and that $\lambda$ is a simple eigenvalue. On the other hand, the assumption $\frac{f(u)}{u} \geq 0$ implies that

$$
\inf \sigma\left(L_{0}\right) \geq \inf \sigma(L)=\lambda .
$$

If $\lambda=\inf \sigma\left(L_{0}\right)$ were true, we could obtain from $\lambda \notin \sigma_{\text {ess }}\left(L_{0}\right)$ that $\lambda$ is also an isolated eigenvalue of $L_{0}$ with a positive eigenfunction $v$. But then, since $f(u) \not \equiv 0$ by assumption,

$$
\lambda=\frac{\int_{\mathbb{R}^{N}}\left(|\nabla v|^{2}+V v^{2}\right)}{\int_{\mathbb{R}^{N}} v^{2}}>\frac{\int_{\mathbb{R}^{N}}\left(|\nabla v|^{2}+(V-f(u) / u) v^{2}\right)}{\int_{\mathbb{R}^{N}} v^{2}} \geq \lambda,
$$

a contradiction. Hence $\lambda<\inf \sigma\left(L_{0}\right)$.

We close this section by introducing the extended Lagrangian

$$
G_{\alpha}: H^{1} \times \mathbb{R} \rightarrow \mathbb{R}, \quad G_{\alpha}(u, \lambda):=\Phi(u)-\frac{\lambda}{2}\left(|u|_{2}^{2}-\alpha\right)=\Phi(u)-\frac{\lambda}{2}(\langle S u, u\rangle-\alpha) .
$$

By definition, $u \in H^{1}$ is a critical point of $J_{\alpha}$ with Lagrange multiplier $\lambda$ if and only if $(u, \lambda)$ is a critical point of $G_{\alpha}$. We endow $H^{1} \times \mathbb{R}$ with the natural scalar product

$$
\langle(u, s),(v, t)\rangle:=\langle u, v\rangle+s t .
$$

The respective gradient of $G_{\alpha}$ is

$$
\nabla G_{\alpha}: H^{1} \times \mathbb{R} \rightarrow H^{1} \times \mathbb{R}, \quad \nabla G_{\alpha}(u, \lambda)=\left(\nabla \Phi(u)-\lambda S u,-\frac{1}{2}\left(|u|_{2}^{2}-\alpha\right)\right) .
$$

Moreover, we have

$$
\mathrm{D}^{2} G_{\alpha}(u, \lambda)[(v, \mu)]=\left(\mathrm{D}^{2} \Phi(u) v-\lambda S v-\mu S u,-\langle S u, v\rangle\right) .
$$


The operator $\mathrm{D}^{2} G_{\alpha}(u, \lambda)$ is known in the literature as the Bordered Hessian of $\Phi$ at $(u, \lambda)$. It has been used extensively in finite dimensional settings to discern local extrema of restricted functionals, see, e.g., $[10,23,27,30,47,49]$. We will use it only in Section 3 below for a gluing procedure respecting an $L^{2}$-constraint.

Although we do not need this property in the present paper, we note that a critical point $u \in H^{1}$ of $J_{\alpha}$ is nondegenerate if and only if $\mathrm{D}^{2} G_{\alpha}(u, \lambda)$ is an isomorphism of $H^{1} \times \mathbb{R}$. The proof is straightforward.

\section{Gluing Bumps with $L^{2}$-Constraint}

This section is devoted to the proof of Theorem 1.2, which we reformulate in the following way for matters of convenience. We continue to use the notation introduced in Section 2.

Theorem 3.1. Assume (H1)-(H3) and fix $\alpha>0$. Given $n \in \mathbb{N}, n \geq 2$, suppose that $\bar{u}$ is a fully nondegenerate critical point of $J_{\alpha / n}$ with Lagrange multiplier $\bar{\lambda}$. Let also $\left(a_{k}\right) \subseteq\left(\mathbb{Z}^{N}\right)^{n}$ be a sequence such that $d\left(a_{k}\right) \rightarrow \infty$ as $k \rightarrow \infty$. Then there exists $k_{0} \in \mathbb{N}$ such that for $k \geq k_{0}$ there exist critical points $u_{k}$ of $J_{\alpha}$ with Lagrange multiplier $\lambda_{k}$. Moreover, we have

$$
\left\|u_{k}-v_{k}\right\| \rightarrow 0 \quad \text { and } \quad\left|\lambda_{k}-\bar{\lambda}\right| \rightarrow 0 \quad \text { as } k \rightarrow \infty, \quad \text { where } v_{k}:=\sum_{i=1}^{n} \mathcal{T}_{a_{k}^{i}} \bar{u} \in H^{1},
$$

and the sequence $\left(u_{k}\right)_{k}$ is uniquely determined by these properties for large $k$. Furthermore, if $\bar{u}$ is a positive function and $f(\bar{u}) \geq 0$ on $\mathbb{R}^{N}, f(\bar{u}) \not \equiv 0$, then $u_{k}$ is positive as well for large $k$.

The remainder of this section is devoted to the proof of this theorem. Let $\alpha>0$, $n \geq 2$, and $\bar{u}, \bar{\lambda}$ be as in the statement of the theorem. Since $\bar{u}$ is nondegenerate and freely nondegenerate, Definition 2.5 and Definition 2.7 imply that

$$
B:=\mathrm{D}^{2} \Phi(\bar{u})-\bar{\lambda} S \in \mathcal{L}\left(H^{1}\right) \text { is an isomorphism }
$$

and that

$$
\text { there exists } z_{\bar{u}} \in H^{1} \text { with }\left(z_{\bar{u}}, \bar{u}\right)_{2} \neq 0 \text { and } B z_{\bar{u}}=S \bar{u} \text {. }
$$

Let $\left(a_{k}\right) \subseteq\left(\mathbb{Z}^{N}\right)^{n}$ be a sequence such that $d\left(a_{k}\right) \rightarrow \infty$ as $k \rightarrow \infty$, and let $v_{k} \in H^{1}$ be given as in (3.1) for $k \in \mathbb{N}$. For simplicity we assume that

$$
a_{k}^{1}=0 \quad \text { for all } k \in \mathbb{N} .
$$

We wish to prove that

$$
\nabla G_{\alpha}\left(v_{k}, \bar{\lambda}\right) \rightarrow 0 \quad \text { as } k \rightarrow \infty
$$

and that

$\mathrm{D}^{2} G_{\alpha}\left(v_{k}, \bar{\lambda}\right) \in \mathcal{L}\left(H^{1} \times \mathbb{R}\right)$ is invertible for large $k$, and the norm of the inverse remains bounded as $k \rightarrow \infty$. 
Once these assertions are proved, we may apply Corollary 2.2 with $h:=\nabla G_{\alpha}$ to find, for $k$ large, critical points $u_{k}$ of $J_{\alpha}$ with Lagrange multiplier $\lambda_{k}$ such that (3.1) holds true. Here we use the fact that the sequence $\left(v_{k}\right)_{k}$ is bounded in $H^{1}$ and that $\mathrm{D}^{2} \Phi$ is uniformly continuous on bounded subsets of $H^{1}$.

By the BL-splitting properties, (2.13) implies

$$
\left\|\nabla G_{\alpha}\left(v_{k}, \bar{\lambda}\right)-\sum_{i=1}^{n} \nabla G_{\alpha / n}\left(\mathcal{T}_{a_{k}^{i}} \bar{u}, \bar{\lambda}\right)\right\|_{\mathcal{L}\left(H^{1} \times \mathbb{R}\right)} \rightarrow 0 .
$$

Since $\left\|\nabla G_{\alpha / n}\left(\mathcal{T}_{a_{k}^{i}} \bar{u}, \bar{\lambda}\right)\right\|_{\mathcal{L}\left(H^{1} \times \mathbb{R}\right)}=\left\|\nabla G_{\alpha / n}(\bar{u}, \bar{\lambda})\right\|_{\mathcal{L}\left(H^{1} \times \mathbb{R}\right)}=0$ for $i=1,2, \ldots, n$ and every $k,(3.5)$ follows.

We now turn to the (more difficult) proof of (3.6). For this we consider the operators

$$
B_{k}:=\mathrm{D}^{2} \Phi\left(v_{k}\right)-\bar{\lambda} S \in \mathcal{L}\left(H^{1}\right) \quad \text { for } k \in \mathbb{N} \text {. }
$$

and we claim that

$$
\mathcal{T}_{-a_{k}^{i}} B_{k} \mathcal{T}_{a_{k}^{i}} w \rightarrow B w \quad \text { in } H^{1} \text { for } w \in H^{1}, i=1,2, \ldots, n
$$

To see this, we recall that $\mathrm{D}^{2} \Psi$ BL-splits and that therefore

$$
\mathrm{D}^{2} \Psi\left(v_{k}\right)=\sum_{j=1}^{n} \mathrm{D}^{2} \Psi\left(\mathcal{T}_{a_{k}^{j}} \bar{u}\right)+o(1) \quad \text { in } \mathcal{L}\left(H^{1}\right)
$$

which implies that

$$
B_{k}=I-\bar{\lambda} S-\mathrm{D}^{2} \Psi\left(v_{k}\right)=I-\bar{\lambda} S-\sum_{j=1}^{n} \mathrm{D}^{2} \Psi\left(\mathcal{T}_{a_{k}^{j}} \bar{u}\right)+o(1) \quad \text { in } \mathcal{L}\left(H^{1}\right) .
$$

It is easy to see that

$$
\mathcal{T}_{-a_{k}^{i}} \mathrm{D}^{2} \Psi\left(\mathcal{T}_{a_{k}^{i}} \bar{u}\right) \mathcal{T}_{a_{k}^{i}}=\mathrm{D}^{2} \Psi(\bar{u}) \quad \text { for } k \in \mathbb{N} \text { and } i=1, \ldots, n
$$

Moreover, if $i \neq j$, then for $w \in H^{1}$ we have

$$
\mathrm{D}^{2} \Psi\left(\mathcal{T}_{a_{k}^{j}} \bar{u}\right) \mathcal{T}_{a_{k}^{i}} w=\mathcal{T}_{a_{k}^{j}} \mathcal{T}_{-a_{k}^{j}} \mathrm{D}^{2} \Psi\left(\mathcal{T}_{a_{k}^{j}} \bar{u}\right) \mathcal{T}_{a_{k}^{j}} \mathcal{T}_{a_{k}^{i}-a_{k}^{j}} w=\mathcal{T}_{a_{k}^{j}} \mathrm{D}^{2} \Psi(\bar{u}) \mathcal{T}_{a_{k}^{i}-a_{k}^{j}} w \rightarrow 0
$$

in $H^{1}$, since $\mathcal{T}_{a_{k}^{i}-a_{k}^{j}} w \rightarrow 0$ and $\mathrm{D}^{2} \Psi(\bar{u}) \in \mathcal{L}\left(H^{1}\right)$ is a compact operator. Combining (3.9)-(3.11) and recalling that $S$ commutes with $\mathcal{T}_{a_{k}^{i}}$, we find that

$$
\begin{aligned}
\mathcal{T}_{-a_{k}^{i}} B_{k} \mathcal{T}_{a_{k}^{i}} w & =(I-\bar{\lambda} S) w-\sum_{j=1}^{n} \mathcal{T}_{-a_{k}^{i}} \mathrm{D}^{2} \Psi\left(\mathcal{T}_{a_{k}^{j}} \bar{u}\right) \mathcal{T}_{a_{k}^{i}} w+o(1) \\
& =(I-\bar{\lambda} S) w-\mathrm{D}^{2} \Psi(\bar{u}) w+o(1)=B w+o(1) \quad \text { as } k \rightarrow \infty
\end{aligned}
$$

for $w \in H^{1}$ and $i=1, \ldots, n$, as claimed in (3.7). 
We note that (3.7) implies that

$$
\mathcal{T}_{-a_{k}^{i}} B_{k} \mathcal{T}_{a_{k}^{j}} w=\mathcal{T}_{a_{k}^{j}-a_{k}^{i}} \mathcal{T}_{-a_{k}^{j}} B_{k} \mathcal{T}_{a_{k}^{j}} w=\mathcal{T}_{a_{k}^{j}-a_{k}^{i}} B w+o(1) \rightarrow 0 \quad \text { in } H^{1}
$$

for $w \in H^{1}$ and $i \neq j$. We now prove (3.6) by contradiction. Supposing that (3.6) does not hold true, we find, after passing to a subsequence, that there are $w_{k} \in H^{1}$ and $\mu_{k} \in \mathbb{R}$ such that $\left\|w_{k}\right\|^{2}+\mu_{k}^{2}=1$ and $\mathrm{D}^{2} G_{\alpha}\left(v_{k}, \bar{\lambda}\right)\left[\left(w_{k}, \mu_{k}\right)\right] \rightarrow 0$. By (2.14) this implies

$$
B_{k} w_{k}-\mu_{k} S v_{k} \rightarrow 0 \quad \text { in } H^{1}
$$

and

$$
\left(v_{k}, w_{k}\right)_{2} \rightarrow 0 \quad \text { in } \mathbb{R}
$$

Define for $i=1,2, \ldots, n$, possibly after passing to a subsequence, the functions

$$
w^{i}:=\underset{k \rightarrow \infty}{\mathrm{W}-\lim } \mathcal{T}_{-a_{k}^{i}} w_{k} \in H^{1}
$$

and $\mu:=\lim _{k \rightarrow \infty} \mu_{k}$. Let $z_{\bar{u}} \in H^{1}$ be given as in (3.3). Forming the $H^{1}$-scalar product of (3.13) with $\mathcal{T}_{a_{k}^{i}} z_{\bar{u}}$ and using (3.7) together with the fact that $\mathcal{T}_{-a_{k}^{i}} v_{k} \rightarrow \bar{u}$ in $H^{1}$, we obtain that

$$
\begin{aligned}
o(1) & =\left\langle B_{k} w_{k}, \mathcal{T}_{a_{k}^{i}} z_{\bar{u}}\right\rangle-\mu_{k}\left\langle S v_{k}, \mathcal{T}_{a_{k}^{i}} z_{\bar{u}}\right\rangle=\left\langle w_{k}, B_{k} \mathcal{T}_{a_{k}^{i}} z_{\bar{u}}\right\rangle-\mu_{k}\left(v_{k}, \mathcal{T}_{a_{k}^{i}} z_{\bar{u}}\right)_{2} \\
& =\left\langle\mathcal{T}_{-a_{k}^{i}} w_{k}, \mathcal{T}_{-a_{k}^{i}} B_{k} \mathcal{T}_{a_{k}^{i}} z_{\bar{u}}\right\rangle-\mu_{k}\left(\mathcal{T}_{-a_{k}^{i}} v_{k}, z_{\bar{u}}\right)_{2}=\left\langle w^{i}, B z_{\bar{u}}\right\rangle-\mu\left(\bar{u}, z_{\bar{u}}\right)_{2}+o(1) \\
& =\left\langle w^{i}, S \bar{u}\right\rangle-\mu\left(\bar{u}, z_{\bar{u}}\right)_{2}+o(1)=\left(w^{i}, \bar{u}\right)_{2}-\mu\left(\bar{u}, z_{\bar{u}}\right)_{2}+o(1)
\end{aligned}
$$

for $i=1, \ldots, n$. Hence

$$
\left(w^{i}, \bar{u}\right)_{2}=\mu\left(\bar{u}, z_{\bar{u}}\right)_{2} \quad \text { for } i=1, \ldots, n .
$$

By (3.14) we thus have that

$0=\lim _{k \rightarrow \infty}\left(v_{k}, w_{k}\right)_{2}=\lim _{k \rightarrow \infty} \sum_{i=1}^{n}\left(\mathcal{T}_{a_{k}^{i}} \bar{u}, w_{k}\right)_{2}=\lim _{k \rightarrow \infty} \sum_{i=1}^{n}\left(\bar{u}, \mathcal{T}_{-a_{k}^{i}} w_{k}\right)_{2}=\sum_{i=1}^{n}\left(\bar{u}, w^{i}\right)_{2}=n \mu\left(\bar{u}, z_{\bar{u}}\right)_{2}$.

Since $\left(\bar{u}, z_{\bar{u}}\right)_{2} \neq 0$, this gives $\mu=0$. Hence (3.13) reduces to

$$
B_{k} w_{k} \rightarrow 0 \quad \text { in } H^{1} \text { as } k \rightarrow \infty \text {. }
$$

We now set

$$
z_{k}:=w_{k}-\sum_{j=1}^{n} \mathcal{T}_{a_{k}^{j}} w^{j} \quad \text { for } k \in \mathbb{N}
$$

SO

$$
\mathcal{T}_{-a_{k}^{i}} z_{k} \rightarrow 0 \quad \text { for } i=1, \ldots, n
$$


By (3.7), (3.12) and (3.15) we have

$$
\begin{aligned}
0=\underset{k \rightarrow \infty}{\mathrm{w}-\lim } \mathcal{T}_{-a_{k}^{i}} B_{k} w_{k} & =\underset{k \rightarrow \infty}{\mathrm{w}-\lim _{k \rightarrow 1}}\left[\sum_{j=1}^{n} \mathcal{T}_{-a_{k}^{i}} B_{k} \mathcal{T}_{a_{k}^{j}} w^{j}+\mathcal{T}_{-a_{k}^{i}} B_{k} z_{k}\right] \\
& =B w^{i}+\underset{k \rightarrow \infty}{\mathrm{w}-\lim } \mathcal{T}_{-a_{k}^{i}} B_{k} z_{k} .
\end{aligned}
$$

Moreover,

$$
\mathrm{D}^{2} \Psi(\bar{u}) \mathcal{T}_{-a_{k}^{i}} z_{k} \rightarrow 0 \quad \text { in } H^{1} \text { for } i=1, \ldots, n
$$

by (3.16) and since $\mathrm{D}^{2} \Psi(\bar{u}) \in \mathcal{L}\left(H^{1}\right)$ is a compact operator, which by (3.10) implies that

$$
\mathcal{T}_{-a_{k}^{i}} \mathrm{D}^{2} \Psi\left(\mathcal{T}_{a_{k}^{j}} \bar{u}\right) z_{k}=\mathcal{T}_{a_{k}^{j}-a_{k}^{i}} \mathrm{D}^{2} \Psi(\bar{u}) \mathcal{T}_{-a_{k}^{j}} z_{k} \rightarrow 0 \quad \text { in } H^{1}
$$

for $i, j=1, \ldots, n$. Using (3.9) again, we obtain

$$
\begin{aligned}
\underset{k \rightarrow \infty}{\mathrm{W}-\lim } \mathcal{T}_{-a_{k}^{i}} B_{k} z_{k} & =\underset{k \rightarrow \infty}{\mathrm{w}-\lim }\left(\mathcal{T}_{-a_{k}^{i}}(I-\bar{\lambda} S) z_{k}-\sum_{j=1}^{n} \mathcal{T}_{-a_{k}^{i}} \mathrm{D}^{2} \Psi\left(\mathcal{T}_{a_{k}^{j}} \bar{u}\right) z_{k}\right) \\
& =\underset{k \rightarrow \infty}{\mathrm{w}-\lim _{k}}(I-\bar{\lambda} S) \mathcal{T}_{-a_{k}^{i}} z_{k}=0
\end{aligned}
$$

for $i=1, \ldots, n$. Combining this with (3.17), we conclude that $B w^{i}=0$ for $i=1, \ldots, n$ and thus

$$
w^{i}=0 \quad \text { for } i=1, \ldots, n
$$

by (3.2). We therefore have $w_{k}=z_{k}$ for all $k$. Recalling (3.15), (3.9), (3.4), and choosing $i=1$ in (3.18) and (3.19), we find

$$
\begin{aligned}
o(1)=B_{k} w_{k}=B_{k} z_{k} & =(I-\bar{\lambda} S) z_{k}-\sum_{j=1}^{n} \mathrm{D}^{2} \Psi\left(\mathcal{T}_{a_{k}^{j}} \bar{u}\right) z_{k}+o(1)=(I-\bar{\lambda} S) z_{k}+o(1) \\
& =(I-\bar{\lambda} S) z_{k}-\mathrm{D}^{2} \Psi(\bar{u}) z_{k}+o(1)=B z_{k}+o(1)=B w_{k}+o(1) .
\end{aligned}
$$

and thus $w_{k} \rightarrow 0$ in $H^{1}$ by (3.2). Since $\mu=0$, this contradicts our assumption that $\left\|w_{k}\right\|^{2}+\mu_{k}^{2}=1$ for all $k$. This proves (3.6), as desired.

In the following we assume $N \geq 3$. The cases $N=1,2$ are proved similarly, ignoring those terms below that include the critical exponent $2^{*}$.

As remarked above, applying Corollary 2.2 with $h:=\nabla G_{\alpha}$ now yields, for $k$ large, critical points $u_{k}$ of $J_{\alpha}$ with Lagrange multiplier $\lambda_{k}$ such that (3.1) holds true. To finish the proof of Theorem 3.1, we now assume that $\bar{u} \in H^{1}$ is positive with $f(\bar{u}) \geq 0$ in $\mathbb{R}^{N}$, $f(\bar{u}) \not \equiv 0$, and we show that $u_{k}$ is also positive for $k$ large. By Lemma 2.9 we then have $\bar{\lambda}<\inf \sigma(-\Delta+V)=\gamma$, so

$$
\int_{\mathbb{R}^{N}}\left(|\nabla v|^{2}+[V-\bar{\lambda}]|v|^{2}\right) \geq(\gamma-\bar{\lambda})\|v\|^{2} \quad \text { for all } v \in H^{1} .
$$

On the other hand, for fixed $\varepsilon \in(0, \gamma-\bar{\lambda})$ it easily follows from (H3), Sobolev embeddings, the representation (2.3), and (2.4), that there is a constant $C>0$ such that

$$
\int_{\mathbb{R}^{N}} f(v) v \leq \varepsilon\|v\|^{2}+C\|v\|^{p}+\varepsilon\|v\|^{2^{*}} \quad \text { for } v \in H^{1} .
$$


Moreover, since $v_{k}$ is positive, (3.1) implies that $u_{k}^{-}:=\min \left\{u_{k}, 0\right\} \rightarrow 0$ in $H^{1}$ as $k \rightarrow \infty$. However, we have

$$
\begin{aligned}
0 & =\int_{\mathbb{R}^{N}}\left(-\Delta u_{k}+\left[V-\lambda_{k}\right] u_{k}-f\left(u_{k}\right)\right) u_{k}^{-} \\
& =\int_{\mathbb{R}^{N}}\left(\left|\nabla u_{k}^{-}\right|^{2}+\left[V-\lambda_{k}\right]\left|u_{k}^{-}\right|^{2}\right)-\int_{\mathbb{R}^{N}} f\left(u_{k}^{-}\right) u_{k}^{-}
\end{aligned}
$$

and therefore

$$
\begin{aligned}
(\gamma-\bar{\lambda})\left\|u_{k}^{-}\right\|^{2} & \leq \int_{\mathbb{R}^{N}}\left(\left|\nabla u_{k}^{-}\right|^{2}+[V-\bar{\lambda}]\left|u_{k}^{-}\right|^{2}\right) \\
& =o(1)\left|u_{k}^{-}\right|_{2}^{2}+\int_{\mathbb{R}^{N}}\left(\left|\nabla u_{k}^{-}\right|^{2}+\left[V-\lambda_{k}\right]\left|u_{k}^{-}\right|^{2}\right) \\
& =o(1)\left\|u_{k}^{-}\right\|^{2}+\int_{\mathbb{R}^{N}} f\left(u_{k}^{-}\right) u_{k}^{-} \\
& \leq(\varepsilon+o(1))\left\|u_{k}^{-}\right\|^{2}+C\left\|u_{k}^{-}\right\|^{p}+\varepsilon\left\|u_{k}^{-}\right\|^{2^{*}}
\end{aligned}
$$

By the choice of $\varepsilon$, this implies that $u_{k}^{-}=0$ for large $k$. Consequently, $u_{k}$ is strictly positive on $\mathbb{R}^{N}$ for large $k$ by the strong maximum principle. The proof of Theorem 3.1 is finished.

\section{Morse Index and nondegeneracy of normalized multibump solutions}

In this section, we prove a general result on the nondegeneracy and the Morse index of normalized multibump solutions built from fully nondegenerate critical points of the restriction of $\Phi$ to $\Sigma_{\alpha / n}$. Moreover, we also complete the proof of Corollary 1.4 at the end of the section.

Recall, for $\alpha>0$ and a critical point $u$ of $J_{\alpha}=\left.\Phi\right|_{\Sigma_{\alpha}}$, the definitions of the Morse index $m(u)$ and the free Morse index $m_{\mathrm{f}}(u)$ given in Definition 2.5. The following theorem is the main result of this section, and together with Lemma 2.6 it readily implies Theorem 1.3.

Theorem 4.1. Assume (H1)-(H3) and fix $\alpha>0$. Given $n \in \mathbb{N}, n \geq 2$, suppose that $\bar{u}$ is a fully nondegenerate critical point of $J_{\alpha / n}$ with Lagrange multiplier $\bar{\lambda}$ and finite Morse index $m(\bar{u})$. Furthermore, let $\left(a_{k}\right) \subseteq\left(\mathbb{Z}^{N}\right)^{n}$ be a sequence such that $d\left(a_{k}\right) \rightarrow \infty$ as $k \rightarrow \infty$, and such that the critical points $u_{k}$ of $J_{\alpha}$ with Lagrange multiplier $\lambda_{k}$ and with

(4.1) $\left\|u_{k}-v_{k}\right\| \rightarrow 0 \quad$ and $\quad\left|\lambda_{k}-\bar{\lambda}\right| \rightarrow 0 \quad$ as $k \rightarrow \infty, \quad$ where $v_{k}:=\sum_{i=1}^{n} \mathcal{T}_{a_{k}^{i}} \bar{u} \in H^{1}$

from Theorem 3.1 exist for all $k$. Then, for $k$ sufficiently large, $u_{k}$ is a nondegenerate critical point of $J_{\alpha}, m\left(u_{k}\right)=n(m(\bar{u})+1)-1$ if $\left(\bar{u}, z_{\bar{u}}\right)_{2}<0$, and $m\left(u_{k}\right)=n m(\bar{u})$ if $\left(\bar{u}, z_{\bar{u}}\right)_{2}>0$. If (H4) holds true, then $m\left(u_{k}\right)>0$ for large $k$. 
To prove this Theorem, we set $B:=\mathrm{D}^{2} \Phi(\bar{u})-\bar{\lambda} S$ and $B_{k}:=\mathrm{D}^{2} \Phi\left(v_{k}\right)-\bar{\lambda} S$, as in Section 3. Moreover, we consider the self adjoint operators

$$
C_{k}:=\mathrm{D}^{2} \Phi\left(u_{k}\right)-\lambda_{k} S \in \mathcal{L}\left(H^{1}\right)
$$

for $k \in \mathbb{N}$. First we show that the constrained critical points $u_{k}$ of $\Phi$ are freely nondegenerate and that

$$
m_{\mathrm{f}}\left(u_{k}\right)=n m_{\mathrm{f}}(\bar{u}) \quad \text { for large } k .
$$

To this end it is sufficient to prove the following

Lemma 4.2. It holds true that

$$
\limsup _{k \rightarrow \infty} \inf _{\substack{W \leqslant H^{1} \\ \operatorname{dim} W=n m_{\mathrm{f}}(\bar{u})}} \sup _{\substack{w \in W \\\|w\|=1}}\left\langle C_{k} w, w\right\rangle<0
$$

and

$$
\liminf _{k \rightarrow \infty} \inf _{\substack{W \leqslant H^{1} \\ \operatorname{dim} W=n m_{\mathrm{f}}(\bar{u})+1}} \sup _{\substack{w \in W \\\|w\|=1}}\left\langle C_{k} w, w\right\rangle>0 .
$$

Proof. By (4.1) and since $\mathrm{D}^{2} \Phi: H^{1} \rightarrow \mathcal{L}\left(H^{1}\right)$ is uniformly continuous on bounded subsets of $H^{1}$, the assertion follows once we have established the following estimates:

$$
\begin{array}{ccc}
\limsup _{k \rightarrow \infty} & \inf _{\substack{W \leqslant H^{1} \\
\operatorname{dim} W=n m_{\mathrm{f}}(\bar{u})}} \sup _{\substack{w \in W \\
\|w\|=1}}\left\langle B_{k} w, w\right\rangle<0, \\
\liminf _{k \rightarrow \infty} \inf _{\substack{W \leqslant H^{1} \\
\operatorname{dim} W=n m_{\mathrm{f}}(\bar{u})+1}} \sup _{\substack{w \in W \\
\|w\|=1}}\left\langle B_{k} w, w\right\rangle>0 .
\end{array}
$$

Let $Z \subset H^{1}$ denote the generalized eigenspace of the self-adjoint operator $B$ in $H^{1}$ corresponding to its $m_{\mathrm{f}}(\bar{u})$ negative eigenvalues. Pick $\delta>0$ such that $\langle B w, w\rangle \leq-\delta\|w\|^{2}$ for all $w \in Z$ and $\langle B y, y\rangle \geq \delta\|y\|^{2}$ for all $y \in Z^{\perp}$. Put

$$
Z_{k}:=\sum_{i=1}^{n} \mathcal{T}_{a_{k}^{i}} Z \subset H^{1} \quad \text { for } k \in \mathbb{N} .
$$

Since $d\left(a_{k}\right) \rightarrow \infty$, the sum is direct and hence $\operatorname{dim} Z_{k}=n m_{\mathrm{f}}(\bar{u})$ for $k$ sufficiently large. If $w_{k} \in Z_{k}$ satisfies $\left\|w_{k}\right\|=1$ for all $k$, then it suffices to show

$$
\limsup _{k \rightarrow \infty}\left\langle B_{k} w_{k}, w_{k}\right\rangle \leq-\delta
$$

along a subsequence to prove (4.4). We write

$$
w_{k}=\sum_{i=1}^{n} \mathcal{T}_{a_{k}^{i}} \rho_{k}^{i} \quad \text { for } k \in \mathbb{N} \text { with } \rho_{k}^{i} \in Z .
$$

Since $Z$ is finite dimensional, we may pass to a subsequence such that $\rho_{k}^{i} \rightarrow \rho^{i} \in Z$ for $i=1, \ldots, n$ as $k \rightarrow \infty$. It is easy to see that then

$$
1=\left\|w_{k}\right\|^{2}=\sum_{i=1}^{n}\left\|\rho^{i}\right\|^{2}+o(1) \quad \text { as } k \rightarrow \infty .
$$


Thus (3.7) and (3.12) imply that

$$
\begin{aligned}
\left\langle B_{k} w_{k}, w_{k}\right\rangle=\sum_{i, j=1}^{n}\left\langle B_{k} \mathcal{T}_{a_{k}^{i}} \rho_{k}^{i}, \mathcal{T}_{a_{k}^{j}} \rho_{k}^{j}\right\rangle & =\sum_{i, j=1}^{n}\left\langle\mathcal{T}_{-a_{k}^{j}} B_{k} \mathcal{T}_{a_{k}^{i}} \rho^{i}, \rho^{j}\right\rangle+o(1)=\sum_{i=1}^{n}\left\langle B \rho^{i}, \rho^{i}\right\rangle+o(1) \\
& \leq-\delta \sum_{i=1}^{n}\left\|\rho^{i}\right\|^{2}+o(1)=-\delta+o(1)
\end{aligned}
$$

that is, (4.6).

If $y_{k} \in Z_{k}^{\perp}$ satisfies $\left\|y_{k}\right\|=1$ for all $k$, then it suffices to show

$$
\liminf _{k \rightarrow \infty}\left\langle B_{k} y_{k}, y_{k}\right\rangle \geq \delta
$$

for a subsequence to prove (4.5). Passing to a subsequence, we may assume that

$$
w^{i}:=\underset{k \rightarrow \infty}{\mathrm{w}-\lim } \mathcal{T}_{-a_{k}^{i}} y_{k}
$$

exists for $i=1, \ldots, n$. Let $v \in Z$. Since $\mathcal{T}_{a_{k}^{i}} v \in Z_{k}$, we infer that

$$
0=\left\langle\mathcal{T}_{a_{k}^{i}} v, y_{k}\right\rangle=\left\langle v, \mathcal{T}_{-a_{k}^{i}} y_{k}\right\rangle=\left\langle v, w^{i}\right\rangle+o(1) \quad \text { for } i=1, \ldots, n .
$$

Consequently,

$$
w^{i} \in Z^{\perp} \quad \text { for } i=1, \ldots, n .
$$

We now set

$$
z_{k}:=y_{k}-\sum_{i=1}^{n} \mathcal{T}_{a_{k}^{i}} w^{i} \quad \text { for } k \in \mathbb{N}
$$

noting that

$$
\underset{k \rightarrow \infty}{\mathrm{W}-\lim } \mathcal{T}_{-a_{k}^{i}} z_{k}=0 \quad \text { for } i=1, \ldots, n .
$$

In particular, this implies that

$$
z_{k} \rightarrow 0 \quad \text { in } H^{1}
$$

by (3.4) which we may again assume without loss of generality. Using (3.7), (3.12), and (4.9) we obtain the splitting

$$
\begin{aligned}
\left\langle B_{k} y_{k}, y_{k}\right\rangle & =\left\langle B_{k} z_{k}, z_{k}\right\rangle+2 \sum_{i=1}^{n}\left\langle B_{k} \mathcal{T}_{a_{k}^{i}} w^{i}, z_{k}\right\rangle+\sum_{i, j=1}^{n}\left\langle B_{k} \mathcal{T}_{a_{k}^{i}} w^{i}, \mathcal{T}_{a_{k}^{j}} w^{j}\right\rangle \\
& =\left\langle B_{k} z_{k}, z_{k}\right\rangle+2 \sum_{i=1}^{n}\left\langle\mathcal{T}_{-a_{k}^{i}} B_{k} \mathcal{T}_{a_{k}^{i}} w^{i}, \mathcal{T}_{-a_{k}^{i}} z_{k}\right\rangle+\sum_{i, j=1}^{n}\left\langle\mathcal{T}_{-a_{k}^{j}} B_{k} \mathcal{T}_{a_{k}^{i}} w^{i}, w^{j}\right\rangle \\
& =\left\langle B_{k} z_{k}, z_{k}\right\rangle+\sum_{i=1}^{n}\left\langle B w^{i}, w^{i}\right\rangle+o(1)
\end{aligned}
$$


where

$$
\begin{aligned}
\left\langle B_{k} z_{k}, z_{k}\right\rangle & =\left\|z_{k}\right\|^{2}-\lambda\left|z_{k}\right|_{2}^{2}-\left\langle\mathrm{D}^{2} \Psi\left(v_{k}\right) z_{k}, z_{k}\right\rangle \\
& =\left\|z_{k}\right\|^{2}-\lambda\left|z_{k}\right|_{2}^{2}-\sum_{i=1}^{n}\left\langle\mathrm{D}^{2} \Psi\left(\mathcal{T}_{a_{k}^{i}} \bar{u}\right) z_{k}, z_{k}\right\rangle+o(1) \\
& =\left\|z_{k}\right\|^{2}-\lambda\left|z_{k}\right|_{2}^{2}-\sum_{i=1}^{n}\left\langle\mathrm{D}^{2} \Psi(\bar{u}) \mathcal{T}_{-a_{k}^{i}} z_{k}, \mathcal{T}_{-a_{k}^{i}} z_{k}\right\rangle+o(1) \\
& =\left\|z_{k}\right\|^{2}-\lambda\left|z_{k}\right|_{2}^{2}+o(1) \\
& =\left\|z_{k}\right\|^{2}-\lambda\left|z_{k}\right|_{2}^{2}-\left\langle\mathrm{D}^{2} \Psi(\bar{u}) z_{k}, z_{k}\right\rangle+o(1) \\
& =\left\langle B z_{k}, z_{k}\right\rangle+o(1) .
\end{aligned}
$$

Here we have used (3.8), (3.10), (4.9), (4.10), and the compactness of the operator $\mathrm{D}^{2} \Psi(\bar{u}) \in \mathcal{L}\left(H^{1}\right)$.

Let $P \in \mathcal{L}\left(\mathcal{H}^{1}\right)$ denote the $\langle\cdot, \cdot\rangle$-orthogonal projection on $Z$, and let $Q:=I-P$. Since $P$ has finite range, we see that

$$
z_{k}-Q z_{k}=P z_{k} \rightarrow 0 \quad \text { in } H^{1} \text { as } k \rightarrow \infty .
$$

Combining (4.8), (4.11), (4.12), and (4.13), we obtain

$$
\begin{aligned}
\left\langle B_{k} y_{k}, y_{k}\right\rangle & =\left\langle B Q z_{k}, Q z_{k}\right\rangle+\sum_{i=1}^{n}\left\langle B w^{i}, w^{i}\right\rangle+o(1) \geq \delta\left(\left\|Q z_{k}\right\|^{2}+\sum_{i=1}^{n}\left\|w^{i}\right\|^{2}\right)+o(1) \\
& =\delta\left(\left\|z_{k}\right\|^{2}+\sum_{i=1}^{n}\left\|w^{i}\right\|^{2}\right)+o(1)=\delta\left\|y_{k}\right\|^{2}+o(1)=\delta+o(1),
\end{aligned}
$$

and hence (4.7).

From Lemma 4.2 it follows that $C_{k}$ is invertible for large $k$ and that the norm of its inverse remains bounded as $k \rightarrow \infty$. We now recall the function $z_{u_{k}}=C_{k}^{-1} S u_{k} \in H^{1}$, which by Lemma 2.6 is of key importance to compute $m\left(u_{k}\right)$.

Lemma 4.3. For $i=1, \ldots, n$ we have that

$$
\mathcal{T}_{-a_{k}^{i}} z_{u_{k}} \rightarrow z_{\bar{u}}=B^{-1} S \bar{u} \quad \text { in } H^{1} \text { as } k \rightarrow \infty .
$$

Proof. Let $\psi \in H^{1}$, and let $\varphi=B^{-1} \psi \in H^{1}$. Recalling that $\mathrm{D}^{2} \Phi: H^{1} \rightarrow \mathcal{L}\left(H^{1}\right)$ is uniformly continuous on bounded subsets of $H^{1}$, we may deduce from (3.7) that

$$
\mathcal{T}_{-a_{k}^{i}} C_{k} \mathcal{T}_{a_{k}^{i}} \varphi=\mathcal{T}_{-a_{k}^{i}} B_{k} \mathcal{T}_{a_{k}^{i}} \varphi+o(1) \rightarrow B \varphi=\psi \quad \text { in } H^{1}
$$

as $k \rightarrow \infty$. Since moreover the sequence $\left(z_{u_{k}}\right)_{k}$ is bounded in $H^{1}$ and $\mathcal{T}_{-a_{k}^{i}} u_{k} \rightarrow \bar{u}$ in $H^{1}$ as $k \rightarrow \infty$, we have that

$$
\begin{aligned}
\left\langle z_{\bar{u}}, \psi\right\rangle & =\left\langle B^{-1}(S \bar{u}), \psi\right\rangle=\langle S \bar{u}, \varphi\rangle=\left\langle S\left(\mathcal{T}_{-a_{k}^{i}} u_{k}\right), \varphi\right\rangle+o(1)=\left\langle S u_{k}, \mathcal{T}_{a_{k}^{i}} \varphi\right\rangle+o(1) \\
& =\left\langle C_{k} z_{u_{k}}, \mathcal{T}_{a_{k}^{i}} \varphi\right\rangle+o(1)=\left\langle z_{u_{k}}, C_{k} \mathcal{T}_{a_{k}^{i}} \varphi\right\rangle+o(1)=\left\langle\mathcal{T}_{-a_{k}^{i}} z_{u_{k}}, \mathcal{T}_{-a_{k}^{i}} C_{k} \mathcal{T}_{a_{k}^{i}} \varphi\right\rangle+o(1) \\
& =\left\langle\mathcal{T}_{-a_{k}^{i}} z_{u_{k}}, \psi\right\rangle+o(1) \quad \text { as } k \rightarrow \infty .
\end{aligned}
$$


We may now complete the

Proof of Theorem 4.1. With the help of Lemma 4.3, we compute

$$
\begin{aligned}
\left(u_{k}, z_{u_{k}}\right)_{2}=\left(v_{k}, z_{u_{k}}\right)_{2}+o(1)=\sum_{i=1}^{n}\left(\mathcal{T}_{a_{k}^{i}} \bar{u}, z_{u_{k}}\right)_{2}+o(1) & =\sum_{i=1}^{n}\left(\bar{u}, \mathcal{T}_{-a_{k}^{i}} z_{u_{k}}\right)_{2}+o(1) \\
& =n\left(\bar{u}, z_{\bar{u}}\right)_{2}+o(1) .
\end{aligned}
$$

Since $\left(\bar{u}, z_{\bar{u}}\right)_{2} \neq 0$ as $\bar{u}$ is fully nondegenerate by assumption, we infer that $\left(u_{k}, z_{u_{k}}\right)_{2}$ is also nonzero and has the same sign as $\left(\bar{u}, z_{\bar{u}}\right)_{2}$ for large $k$. Moreover, $u_{k}$ is freely nondegenerate by Lemma 4.2 , so Lemma 2.6 yields that $u_{k}$ is a fully nondegenerate critical point of $\left.\Phi\right|_{\Sigma_{\alpha}}$ for large $k$. Its Morse index is, by the same token, $m\left(u_{k}\right)=$ $m_{\mathrm{f}}\left(u_{k}\right)-1=n m_{\mathrm{f}}(\bar{u})-1=n(m(\bar{u})+1)-1$ if $\left(\bar{u}, z_{\bar{u}}\right)_{2}<0$, and it is $m\left(u_{k}\right)=m_{\mathrm{f}}\left(u_{k}\right)=$ $n m_{\mathrm{f}}(\bar{u})=n m(\bar{u})$ if $\left(\bar{u}, z_{\bar{u}}\right)_{2}>0$.

To show the last statement of the present theorem, suppose that (H4) is satisfied. Lemma 2.4 implies that $\langle B \bar{u}, \bar{u}\rangle<0$, that is, $m_{\mathrm{f}}(\bar{u})>0$. In any case it follows from the preceding calculations that $m\left(u_{k}\right)>0$ for large $k$. This completes the proof of Theorem 4.1.

We close this section by completing the

Proof of Corollary 1.4. Let $\bar{u}$ be a nondegenerate local minimum of $J_{\alpha / n}$ with Lagrange multiplier $\bar{\lambda}$. Moreover, let $\left(a_{k}\right) \subseteq\left(\mathbb{Z}^{N}\right)^{n}$ be a sequence such that $d\left(a_{k}\right) \rightarrow \infty$ as $k \rightarrow$ $\infty$. By Lemma 2.8, $\bar{u}$ is fully nondegenerate and, without loss of generality, a positive function. Thus, (H4) and Theorem 3.1 imply the existence of positive critical points $u_{k}$ of $J_{\alpha}$ with Lagrange multiplier $\lambda_{k}$ for large $k$ and such that (4.1) holds true. Moreover, the sequence $\left(u_{k}\right)_{k}$ is uniquely determined by these properties. Since moreover $m_{\mathrm{f}}(\bar{u})>$ $0=m(\bar{u})$ by $(\mathrm{H} 4)$ and Lemma 2.4 , Theorem 4.1 now implies that $u_{k}$ is nondegenerate with $m\left(u_{k}\right)=n-1$ for large $k$.

\section{Proof of Theorem 1.5}

In this section we wish to prove Theorem 1.5. For this we will assume hypotheses (H5) and (H6). Without loss of generality we may also assume for the nondegenerate critical point $x_{0}$ of $V$ that

$$
x_{0}=0 \quad \text { and } \quad V\left(x_{0}\right)=1 .
$$

We are then concerned with positive solutions of the singularly perturbed equation

$$
-\varepsilon^{2} \Delta u+V(x) u=|u|^{p-2} u, \quad u \in H^{1},
$$

where $p \in\left(2,2^{*}\right)$. By [26, Theorem 1.1], there exists $\varepsilon_{0}$ and a family of positive single peak solutions $\bar{u}_{\varepsilon}, \varepsilon \in\left(0, \varepsilon_{0}\right)$, of $(5.1)$ which concentrates at $x_{0}=0$. This means that each $\bar{u}_{\varepsilon}$ has only one local maximum, and the rescaled functions

$$
u_{\varepsilon} \in H^{1}, \quad u_{\varepsilon}(x):=\bar{u}_{\varepsilon}(\varepsilon x)
$$


converge, as $\varepsilon \rightarrow 0$, in $H^{1}$ to the unique radial positive solution of the limit equation

$$
-\Delta u_{0}+u_{0}=u_{0}^{p-1} \quad \text { in } \mathbb{R}^{N} .
$$

Moreover, as follows from the uniqueness statement in [26, Theorem 1.1], this convergence property after rescaling determines the solutions $\bar{u}_{\varepsilon}$ uniquely for $\varepsilon>0$ small. In addition, we can assume by [26, Theorem 6.2] that $\bar{u}_{\varepsilon}$ is nondegenerate, i.e., the linear operator

$$
H^{1} \mapsto H^{1}, \quad v \mapsto v-(p-1)\left(-\varepsilon^{2} \Delta+V\right)^{-1} \bar{u}_{\varepsilon}^{p-2} v \quad \text { is an isomorphism }
$$

for $\varepsilon \in\left(0, \varepsilon_{0}\right)$. Here, for $\varepsilon>0$, the operator $-\varepsilon^{2} \Delta+V \in \mathcal{L}\left(H^{1}, H^{-1}\right)$ is understood as the Hilbert space isomorphism $H^{1} \rightarrow H^{-1}$ associated with the scalar product

$$
(u, v) \mapsto \int_{\mathbb{R}^{N}}\left(\varepsilon^{2} \nabla u \cdot \nabla v+V u v\right)
$$

on $H^{1}$ via Riesz's representation theorem. Since $0<\min V \leq \max V<\infty$, this scalar product is equivalent to the standard scalar product on $H^{1}$, which we denote by

$$
\langle u, v\rangle_{H^{1}}:=\int_{\mathbb{R}^{N}}(\nabla u \cdot \nabla v+u v)
$$

We also let $\|\cdot\|_{H^{1}}$ denote the associated norm.

Lemma 5.1. The map $\left(0, \varepsilon_{0}\right) \rightarrow H^{1}, \varepsilon \mapsto \bar{u}_{\varepsilon}$ is continuous.

Proof. For $\varepsilon>0$, let $K(\varepsilon):=-\varepsilon^{2} \Delta+V \in \mathcal{L}\left(H^{1}, H^{-1}\right)$. Then the map $K:(0, \infty) \rightarrow$ $\mathcal{L}\left(H^{1}, H^{-1}\right)$ is continuous. Moreover, since $p$ is subcritical, the nonlinear superposition operator $H^{1} \rightarrow H^{-1}, u \mapsto|u|^{p-2} u$ is of class $C^{1}$. Consequently, the map

$$
h:(0, \infty) \times H^{1} \rightarrow H^{-1}, \quad(\varepsilon, u) \mapsto K(\varepsilon) u-|u|^{p-2} u
$$

is continuous, and continuously differentiable in its second argument. Since $\bar{u}_{\varepsilon}$ is a weak solution of $(5.1)$, we have $h\left(\varepsilon, u_{\varepsilon}\right)=0$. Furthermore, the operator

$$
h_{u}\left(\varepsilon, u_{\varepsilon}\right)=K(\varepsilon)-(p-1)\left|\bar{u}_{\varepsilon}\right|^{p-2} \in \mathcal{L}\left(H^{1}, H^{-1}\right)
$$

is an isomorphism as a consequence of (5.4). Hence the claim follows from the implicit function theorem, see, e.g., [16, Theorem 15.1].

Since the map $\varepsilon \mapsto \bar{u}_{\varepsilon}$ is continuous and

$$
\left|\bar{u}_{\varepsilon}\right|_{2}^{2}=\int_{\mathbb{R}^{N}} \bar{u}_{\varepsilon}^{2}=\varepsilon^{N} \int_{\mathbb{R}^{N}} u_{\varepsilon}^{2}=\varepsilon^{N} \int_{\mathbb{R}^{N}} u_{0}^{2}+o(1)=o(1) \quad \text { as } \varepsilon \rightarrow 0,
$$

the assertions (i)-(iii) of Theorem 1.5 are already verified. The remainder of this section is devoted to the proof of Theorem 1.5(iv). 
For this we first note that the function $u_{\varepsilon} \in H^{1}$ defined in (5.2) satisfies the rescaled equation

$$
-\Delta u_{\varepsilon}+V_{\varepsilon}(x) u_{\varepsilon}=\left|u_{\varepsilon}\right|^{p-2} u_{\varepsilon}, \quad u \in H^{1}
$$

with

$$
V_{\varepsilon}: \mathbb{R}^{N} \rightarrow \mathbb{R}, \quad V_{\varepsilon}(x)=V(\varepsilon x)
$$

Moreover, by (5.4), the linear operator

$$
B^{\varepsilon} \in \mathcal{L}\left(H^{1}\right), \quad B^{\varepsilon} v=v-(p-1)\left(-\Delta+V_{\varepsilon}\right)^{-1} u_{\varepsilon}^{p-2} v \quad \text { is an isomorphism }
$$

for $\varepsilon \in\left(0, \varepsilon_{0}\right)$. We also note that the functions $u_{\varepsilon}$ have uniform exponential decay, i.e., there exist constants $\alpha, C>0$ such that

$$
\left|u_{\varepsilon}(x)\right| \leq C \mathrm{e}^{-\alpha|x|} \quad \text { for all } x \in \mathbb{R}^{N}, \varepsilon \in\left(0, \varepsilon_{0}\right),
$$

see [26, Lemma 4.2.(i)]. Moreover,

$$
u_{\varepsilon} \rightarrow u_{0} \quad \text { in } H^{2}\left(\mathbb{R}^{N}\right) \text { and uniformly in } \mathbb{R}^{N},
$$

see [26, Theorem 4.1 and Lemma 4.2(ii)]. Note that $u_{\varepsilon}$ satisfies [26, Equation (4.1)] with $c_{i, y, \varepsilon}=0$ since it is a solution of (5.6).

We need to recall some properties of the unique radial positive solution $u_{0}$ of the limit equation (5.3) and therefore consider the functional

$$
\Phi_{0}^{*}: H^{1} \rightarrow \mathbb{R}, \quad \Phi_{0}^{*}(u):=\frac{1}{2} \int_{\mathbb{R}^{N}}\left(|\nabla u|^{2}+u^{2}\right)-\frac{1}{p} \int_{\mathbb{R}^{N}}|u|^{p},
$$

It is easy to see that $\mathrm{D}^{2} \Phi_{0}^{*}\left(u_{0}\right) \in \mathcal{L}\left(H^{1}\right)$ has exactly one negative eigenvalue, the value $2-p$, with corresponding eigenspace generated by $u_{0}$. Here, the symbol $\mathrm{D}^{2}$ denotes the derivative of the gradient with respect to the scalar product $\langle\cdot, \cdot\rangle_{H^{1}}$.

Its kernel is spanned by the partial derivatives $\partial_{1} u_{0}, \partial_{2} u_{0}, \ldots, \partial_{N} u_{0}$, see [42, Lemma 4.2(i)]. Letting $\tilde{H}$ denote the $\langle\cdot, \cdot\rangle$-orthogonal complement of $\operatorname{span}\left(\partial_{1} u_{0}, \partial_{2} u_{0}, \ldots, \partial_{N} u_{0}\right)$ in $H^{1}$, we therefore find that the operator

$$
B^{0} \in \mathcal{L}\left(H^{1}\right), \quad B^{0} v=\mathrm{D}^{2} \Phi_{0}^{*}\left(u_{0}\right) v=v-(p-1)[\Delta+1]^{-1} u_{0}{ }^{p-2}
$$

restricts to an isomorphism $\tilde{H} \rightarrow \tilde{H}$. Moreover, $\tilde{H}$ contains all radial functions, so in particular $u_{*}:=[\Delta+1]^{-1} u_{0} \in \tilde{H}$. Consequently, there exists a unique $z_{*} \in \tilde{H}$ with $B^{0} z_{*}=u_{*}$.

Lemma 5.2. We have

$$
\left(z_{*}, u_{0}\right)_{2}=\left(\frac{N}{4}-\frac{1}{p-2}\right)\left|u_{0}\right|_{2}^{2}=\frac{p-(2+4 / N)}{4 N(p-2)}\left|u_{0}\right|_{2}^{2} .
$$


Proof. For $\lambda>0$, consider the function

$$
w_{\lambda} \in H^{1}, \quad w_{\lambda}(x)=\lambda^{\frac{1}{p-2}} u_{0}(\sqrt{\lambda} x) \quad \text { for } x \in \mathbb{R}^{N},
$$

which is the unique radial positive solution of

$$
-\Delta w_{\lambda}+\lambda w_{\lambda}-w_{\lambda}^{p-1}=0 \quad \text { in } \mathbb{R}^{N},
$$

so $w_{1}=u_{0}$. Moreover, consider

$$
\tilde{z} \in H^{1}, \quad \tilde{z}(x)=\left.\frac{\partial}{\partial \lambda}\right|_{\lambda=1} w_{\lambda}(x) .
$$

We claim that $z_{*}=-\tilde{z}$. Indeed, we have $B^{0} \tilde{z}=-u_{*}$ since differentiating (5.11) at $\lambda=1$ yields

$$
-\Delta \tilde{z}+\tilde{z}-(p-1) u_{0}^{p-2} \tilde{z}=-u_{0} \quad \text { in } \mathbb{R}^{N} .
$$

Moreover, $\tilde{z} \in \tilde{H}$ since $\tilde{z}$ is a radial function. By the remarks above, this implies that $z_{*}=-\tilde{z}$. We therefore compute that

$$
\begin{aligned}
\left(z_{*}, u_{0}\right)_{2} & =-\left(\tilde{z}, u_{0}\right)_{2}=-\left.\frac{1}{2} \frac{\mathrm{d}}{\mathrm{d} \lambda}\right|_{\lambda=1}\left|w_{\lambda}\right|_{2}^{2}=-\left.\frac{1}{2} \frac{\mathrm{d}}{\mathrm{d} \lambda}\right|_{\lambda=1}\left(\lambda^{\frac{2}{p-2}} \int_{\mathbb{R}^{N}} u_{0}^{2}(\sqrt{\lambda} x) \mathrm{d} x\right) \\
& =-\left.\frac{1}{2} \frac{\mathrm{d}}{\mathrm{d} \lambda}\right|_{\lambda=1} \lambda^{\frac{2}{p-2}-\frac{N}{2}}\left|u_{0}\right|_{2}^{2}=\frac{1}{2}\left(\frac{N}{2}-\frac{2}{p-2}\right)\left|u_{0}\right|_{2}^{2},
\end{aligned}
$$

as claimed.

Next we collect some properties of the scaled potentials $V_{\varepsilon}, \varepsilon \in\left(0, \varepsilon_{0}\right)$ defined in (5.7). Note that these functions are uniformly bounded and satisfy

$$
\left|V_{\varepsilon}(x)-1\right| \leq c \varepsilon^{2}|x|^{2} \quad \text { for } x \in \mathbb{R}^{N}, \varepsilon \in\left(0, \varepsilon_{0}\right) \text {, with a constant } c>0 \text {. }
$$

We also note that

$$
\lim _{\varepsilon \rightarrow 0} \frac{\partial_{i} V_{\varepsilon}(x)}{\varepsilon^{2}}=\sum_{j=1}^{N} \partial_{i j} V(0) x_{j} \quad \text { locally uniformly in } x \in \mathbb{R}^{N}
$$

for $i=1, \ldots, N$, so

$$
\left|\partial_{i} V_{\varepsilon}(x)\right| \leq c \varepsilon^{2}|x| \quad \text { for } x \in \mathbb{R}^{N}, \varepsilon \in\left(0, \varepsilon_{0}\right) \text {, with a constant } c>0 .
$$

Next we consider

$$
z_{\varepsilon}:=\left[B^{\varepsilon}\right]^{-1}\left(-\Delta+V_{\varepsilon}\right)^{-1} u_{\varepsilon} \in H^{1}, \quad \text { for } \varepsilon \in\left(0, \varepsilon_{0}\right),
$$

where $B^{\varepsilon}$ is defined in (5.8). Hence $z_{\varepsilon}$ is the unique weak solution of

$$
-\Delta z_{\varepsilon}+V_{\varepsilon}(x) z_{\varepsilon}-(p-1) u_{\varepsilon}^{p-2} z_{\varepsilon}=u_{\varepsilon} \quad \text { in } \mathbb{R}^{N} .
$$


We claim that

$$
\left(z_{\varepsilon}, u_{\varepsilon}\right)_{2} \rightarrow\left(z_{*}, u_{0}\right)_{2} \quad \text { as } \varepsilon \rightarrow 0 .
$$

To prove this, we argue by contradiction and suppose that there exists $\delta>0$ and a sequence $\left(\varepsilon_{n}\right)_{n} \in\left(0, \varepsilon_{0}\right)$ such that $\varepsilon_{n} \rightarrow 0$ as $n \rightarrow \infty$ and

$$
\left|\left(z_{n}, u_{n}\right)_{2}-\left(z_{*}, w\right)_{2}\right| \geq \delta \quad \text { for all } n \in \mathbb{N} \text {, where } z_{n}:=z_{\varepsilon_{n}} \text { and } u_{n}:=u_{\varepsilon_{n}} .
$$

We first claim that the sequence $\left(z_{n}\right)_{n}$ is bounded in $H^{1}$. Indeed, if not, we can pass to a subsequence such that $\left\|z_{n}\right\|_{H^{1}}>0$ for all $n$ and $\left\|z_{n}\right\|_{H^{1}} \rightarrow \infty$ as $n \rightarrow \infty$. We then consider $y_{n}:=\frac{z_{n}}{\left\|z_{n}\right\|_{H^{1}}}$, and we may pass to a subsequence such that $y_{n} \rightarrow y$ in $H^{1}$. Since $y_{n}$ is a weak solution of the equation

$$
-\Delta y_{n}+V_{\varepsilon_{n}} y_{n}-(p-1) u_{n}^{p-2} y_{n}=\frac{u_{n}}{\left\|z_{n}\right\|_{H^{1}}} \quad \text { in } \mathbb{R}^{N} \text { for every } n,
$$

we have

$$
\begin{aligned}
\int_{\mathbb{R}^{N}}\left[\nabla y \nabla v+y v-(p-1) u_{0}^{p-2} v\right] & =\lim _{n \rightarrow \infty} \int_{\mathbb{R}^{N}}\left[\nabla y_{n} \nabla v+V_{\varepsilon_{n}} y_{n} v-(p-1) u_{n}^{p-2} y_{n} v\right] \\
& =\lim _{n \rightarrow \infty} \frac{1}{\left\|z_{n}\right\|_{H^{1}}} \int_{\mathbb{R}^{N}} u_{n} v=0 \quad \text { for every } v \in H^{1} .
\end{aligned}
$$

Consequently, $y \in H^{1}$ is a weak solution of $-\Delta y+y-(p-1) u_{0}^{p-2} y=0$ in $\mathbb{R}^{N}$, which means that $B^{0} y=0$. Hence there exist $a_{1}, \ldots, a_{N} \in \mathbb{R}$ with $y=\sum_{i=1}^{N} a_{i} \partial_{i} u_{0}$. Next we note that $\partial_{i} u_{n}$ solves the equation

$$
-\Delta\left(\partial_{i} u_{n}\right)+V_{\varepsilon} \partial_{i} u_{n}+u_{n} \partial_{i} V_{\varepsilon_{n}}-(p-1) u_{n}^{p-2} \partial_{i} u_{n}=0 \quad \text { for } i=1, \ldots, N .
$$

Multiplying this equation with $y_{n}$ and integrating over $\mathbb{R}^{N}$, we obtain by (5.19) that

$$
\int_{\mathbb{R}^{N}} u_{n} y_{n} \partial_{i} V_{\varepsilon_{n}}=-\frac{1}{\left\|z_{n}\right\|_{H^{1}}} \int_{\mathbb{R}^{N}} u_{n} \partial_{i} u_{n}=0 \quad \text { for all } n \in \mathbb{N}
$$

Dividing this equation by $\varepsilon_{n}^{2}$ and passing to the limit, we may then use (5.9), (5.14), (5.15) and Lebegue's Theorem to see that

$$
\begin{aligned}
0 & =\lim _{n \rightarrow \infty} \frac{1}{\varepsilon_{n}^{2}} \int_{\mathbb{R}^{N}} u_{n} y_{n} \partial_{i} V_{\varepsilon_{n}}=\sum_{j=1}^{N} \int_{\mathbb{R}^{N}} \partial_{i j} V(0) x_{j} u_{0}(x) y(x) \mathrm{d} x \\
& =\sum_{\ell, j=1}^{N} a_{\ell} \partial_{i j} V(0) \int_{\mathbb{R}^{N}} x_{j} u_{0}(x) \partial_{\ell} u_{0}(x) \mathrm{d} x=-\frac{\left|u_{0}\right|_{2}^{2}}{2} \sum_{j=1}^{N} a_{j} \partial_{i j} V(0) \quad \text { for } i=1, \ldots, N .
\end{aligned}
$$

Here we have integrated by parts in the last step. Since 0 is a nondegenerate critical point of $V$ by assumption, we conclude that $a_{j}=0$ for $j=1, \ldots, N$ and therefore 
$y=0$. This implies in particular that $\left(y_{n}^{2}\right)$ is bounded in $L^{p / 2}$ and that $y_{n}^{2} \rightarrow 0$ in $L_{\text {loc }}^{p / 2}$. Moreover, $u_{n}^{p-2} \rightarrow u_{0}^{p-2}$ in $L^{p /(p-2)}$. Testing (5.19) with $y_{n}$ we obtain that

$$
\int_{\mathbb{R}^{N}}\left(\left|\nabla y_{n}\right|^{2}+V_{\varepsilon_{n}}\left|y_{n}\right|^{2}\right)=(p-1) \int_{\mathbb{R}^{N}} u_{n}^{p-2}\left|y_{n}\right|^{2}+\frac{1}{\left\|z_{n}\right\|_{H^{1}}} \int_{\mathbb{R}^{N}} u_{n} y_{n} \rightarrow 0
$$

as $n \rightarrow \infty$ and therefore $\left\|y_{n}\right\|_{H^{1}} \rightarrow 0$ as $n \rightarrow \infty$, which is a contradiction. We thus conclude that the sequence $\left(z_{n}\right)_{n}$ is bounded. We may thus pass to a subsequence such that $z_{n} \rightarrow z$ in $H^{1}$. We then have by $(5.16)$

$$
\begin{aligned}
\int_{\mathbb{R}^{N}}\left[\nabla z \nabla v+z v-(p-1) u_{0}^{p-2} v\right] & =\lim _{n \rightarrow \infty} \int_{\mathbb{R}^{N}}\left[\nabla z_{n} \nabla v+V_{\varepsilon_{n}} z_{n} v-(p-1) u_{n}^{p-2} z_{n} v\right] \\
& =\lim _{n \rightarrow \infty} \int_{\mathbb{R}^{N}} u_{n} v=\int_{\mathbb{R}^{N}} u_{0} v \quad \text { for every } v \in H^{1} .
\end{aligned}
$$

Consequently, $z \in H^{1}$ is a weak solution of $-\Delta z+z-(p-1) u_{0}^{p-2} z=u_{0}$ in $\mathbb{R}^{N}$, which means that $B^{0} z=u_{*}$. As a consequence, $B^{0}\left(z-z_{*}\right)=0$, which implies that $z-z_{*} \in \operatorname{span}\left(\partial_{1} u_{0}, \partial_{2} u_{0}, \ldots, \partial_{N} u_{0}\right)$ and therefore $\left(z-z_{*}, u_{0}\right)_{2}=0$. We thus conclude that

$$
\left(z_{n}, u_{n}\right)_{2} \rightarrow\left(z, u_{0}\right)_{2}=\left(z_{*}, u_{0}\right)_{2} \quad \text { as } n \rightarrow \infty,
$$

contrary to (5.18). This shows (5.17), as claimed. Combining (5.17) with Lemma 5.2, we see that for fixed $p \in\left(2,2^{*}\right) \backslash\left\{2+\frac{4}{N}\right\}$, we may take $\varepsilon_{0}>0$ smaller if necessary such that

$$
\left(z_{\varepsilon}, u_{\varepsilon}\right)_{2}<0 \quad \text { if } 2<p<2+\frac{4}{N} \quad \text { and } \quad\left(z_{\varepsilon}, u_{\varepsilon}\right)_{2}>0 \quad \text { if } 2+\frac{4}{N}<p<2^{*}
$$

Moreover, from (5.20) we immediately deduce (1.6) by rescaling. Since $\bar{u}_{\varepsilon}$ is a critical point of $\Phi_{\varepsilon}$, it is also a critical point of $\left.\Phi_{\varepsilon}\right|_{\sum_{\left|\bar{u}_{\varepsilon}\right|_{2}^{2}}}$ with Lagrange multiplier 0, which implies, together with (1.6) and Definition 1.1, that $\bar{u}_{\varepsilon}$ is a fully nondegenerate critical point of $\left.\Phi_{\varepsilon}\right|_{\Sigma_{\left|\bar{u}_{\varepsilon}\right|_{2}^{2}}}$.

To conclude the proof of Theorem 1.5, it remains to compute the Morse index of $\bar{u}_{\varepsilon}$ for $\varepsilon>0$ small. From (1.6) and Lemma 2.6, we deduce that

$$
m\left(\bar{u}_{\varepsilon}\right)=m_{\mathrm{f}}\left(\bar{u}_{\varepsilon}\right)-1 \text { if } 2<p<2+\frac{4}{N} \quad \text { and } m\left(\bar{u}_{\varepsilon}\right)=m_{\mathrm{f}}\left(\bar{u}_{\varepsilon}\right) \text { if } 2+\frac{4}{N}<p<2^{*} .
$$

It therefore suffices to compute the free Morse index $m_{\mathrm{f}}\left(\bar{u}_{\varepsilon}\right)$, which by rescaling is the same as the free Morse index $m_{\mathrm{f}}\left(u_{\varepsilon}\right)$ with respect to the rescaled potential

$$
\Phi_{\varepsilon}^{*}: H^{1} \rightarrow \mathbb{R}, \quad \Phi_{\varepsilon}^{*}(u):=\frac{1}{2} \int_{\mathbb{R}^{N}}\left(|\nabla u|^{2}+V_{\varepsilon} u^{2}\right)-\frac{1}{p} \int_{\mathbb{R}^{N}}|u|^{p} .
$$

More precisely, the equalities in (1.5) follow from (5.21) once we have shown that

$$
m_{\mathrm{f}}\left(u_{\varepsilon}\right)=m_{V}+1 \quad \text { for all } p \in\left(2,2^{*}\right) \text { and } \varepsilon>0 \text { small, }
$$

where $m_{V}$ denotes the number of negative eigenvalues of the Hessian of $V$ at $x_{0}$. The argument is partly contained in the proof of [35, Theorem 2.5]. Nevertheless, since some details are omitted there, we give a complete proof of (5.22) in Appendix A. The proof of Theorem 1.5 is thus finished. 


\section{Orbital instability}

This section is devoted to the proof of Theorem 1.7. To simplify the presentation we only give a proof for the case $N \geq 3$; the cases $N=1,2$ can be treated similarly, slightly modifying the arguments below.

Throughout this section, we consider the special case where the nonlinearity $f$ is odd. We may therefore write it in the form $f(t)=g\left(|t|^{2}\right) t$, where $g \in C([0, \infty)) \cap C^{1}((0, \infty))$ satisfies $g(0)=0$ and

$$
\lim _{s \rightarrow \infty} \frac{g^{\prime}(s)}{s^{\frac{2^{*}}{2}-2}}=0 .
$$

Note that in this case we have

$$
\Phi(u)=\frac{1}{2}\|u\|^{2}-\int_{\mathbb{R}^{N}} G\left(|u|^{2}\right)=\frac{1}{2} \int_{\mathbb{R}^{N}}\left(|\nabla u|^{2}+V|u|^{2}\right)-\int_{\mathbb{R}^{N}} G\left(|u|^{2}\right)
$$

for $u \in H^{1}$ with $G(t)=\frac{1}{2} \int_{0}^{t} g$ for $t \geq 0$. To prove the assertion on orbital instability given in Theorem 1.7, we apply an argument from [19] with some modifications. We identify $\mathbb{C}$ with $\mathbb{R}^{2}$ and write the time-dependent nonlinear Schrödinger equation (1.8) as the following system in $\mathbf{u}=\left(\begin{array}{l}u_{1} \\ u_{2}\end{array}\right)$ with $u_{1}=\operatorname{Re} u, u_{2}=\operatorname{Im} u$ :

$$
\mathbf{u}_{t}=J\left(-\Delta \mathbf{u}+V(x) \mathbf{u}-g\left(u_{1}^{2}+u_{2}^{2}\right) \mathbf{u}\right) \quad \text { with } \quad J:=\left(\begin{array}{cc}
0 & -1 \\
1 & 0
\end{array}\right) .
$$

In order to set up the functional analytic equation for this system, we denote the dual paring between $H^{-1}$ and $H^{1}$ by $\langle\cdot, \cdot\rangle_{*}$. We put $\mathcal{H}:=H^{1} \times H^{1}$ and write $\mathcal{H}^{*}=H^{-1} \times H^{-1}$ for the topological dual of $\mathcal{H}$. Recalling that we are assuming $\min \sigma(-\Delta+V)>0$, we use the scalar product

$$
\langle u, v\rangle_{\mathcal{H}}=\left\langle u_{1}, v_{1}\right\rangle+\left\langle u_{2}, v_{2}\right\rangle=\sum_{i=1}^{2} \int_{\mathbb{R}^{N}}\left(\nabla u_{i} \cdot \nabla v_{i}+V u_{i} v_{i}\right), \quad \text { for } u, v \in \mathcal{H}
$$

and denote the induced norm by $\|\cdot\|_{\mathcal{H}}$. The dual pairing between $\mathcal{H}^{*}$ and $\mathcal{H}$ is given by

$$
\langle\mathbf{u}, \mathbf{v}\rangle_{\mathcal{H}^{*}, \mathcal{H}}=\left\langle u_{1}, v_{1}\right\rangle_{*}+\left\langle u_{2}, v_{2}\right\rangle_{*} \quad \text { for } \mathbf{u}=\left(\begin{array}{l}
u_{1} \\
u_{2}
\end{array}\right) \in \mathcal{H}^{*}, \mathbf{v}=\left(\begin{array}{l}
v_{1} \\
v_{2}
\end{array}\right) \in \mathcal{H} \text {. }
$$

As usual in the context of Gelfand triples, we consider the continuous embedding $I: H^{1} \hookrightarrow H^{-1}$ given by

$$
\langle I u, v\rangle_{*}:=\int_{\mathbb{R}^{N}} u v \quad \text { for } u, v \in H^{1} .
$$

The corresponding embedding $\mathcal{H} \hookrightarrow \mathcal{H}^{*}$ will also be denoted by $I$, i.e., we set

$$
\langle I \mathbf{u}, \mathbf{v}\rangle_{\mathcal{H}^{*}, \mathcal{H}}:=\int_{\mathbb{R}^{N}}\left(u_{1} v_{1}+u_{2} v_{2}\right) \quad \text { for } \mathbf{u}=\left(\begin{array}{l}
u_{1} \\
u_{2}
\end{array}\right), \mathbf{v}=\left(\begin{array}{l}
v_{1} \\
v_{2}
\end{array}\right) \in \mathcal{H}
$$


With this notation, we write system (6.1) in the more abstract form of a Hamiltonian system. For this we consider the functionals

$$
\tilde{\Phi} \in C^{2}(\mathcal{H}, \mathbb{R}), \quad \tilde{\Phi}(\mathbf{u})=\frac{1}{2}\|\mathbf{u}\|_{\mathcal{H}}^{2}-\int_{\mathbb{R}^{N}} G\left(u_{1}^{2}+u_{2}^{2}\right)
$$

and

$$
\tilde{\Phi}_{\lambda} \in C^{2}(\mathcal{H}, \mathbb{R}), \quad \tilde{\Phi}_{\lambda}(\mathbf{u})=\Phi(\mathbf{u})-\frac{\lambda}{2} \int_{\mathbb{R}^{N}}|\mathbf{u}|^{2} .
$$

With this notation, (6.1) writes as

$$
(I \mathbf{u})_{t}=J \mathrm{~d} \tilde{\Phi}(\mathbf{u}) \quad \text { in } \mathcal{H}^{*}
$$

where $\mathrm{d} \tilde{\Phi}: \mathcal{H} \rightarrow \mathcal{H}^{*}$ denotes the derivative of $\tilde{\Phi}$ and $J$ is regarded as a matrix multiplication operator on $\mathcal{H}^{*}=H^{-1} \times H^{-1}$.

Now let $\varphi \in \Sigma_{\alpha}$ satisfy the assumptions of Theorem 1.7 , and let $\lambda \in \mathbb{R}$ be the corresponding Lagrangian multiplier. Moreover, in the following, we let $\mathrm{d}^{2} \tilde{\Phi}_{\lambda}(\psi) \in \mathcal{L}\left(\mathcal{H}, \mathcal{H}^{*}\right)$ denote the second derivative of $\tilde{\Phi}_{\lambda}$ at $\psi:=\left(\begin{array}{l}\varphi \\ 0\end{array}\right) \in \mathcal{H}$, which by direct computation is given as

$$
\mathrm{d}^{2} \tilde{\Phi}_{\lambda}(\psi)=\left(\begin{array}{cc}
L_{1} & 0 \\
0 & L_{2}
\end{array}\right), \quad \text { where } \quad\left\{\begin{array}{l}
L_{1} w=-\Delta w+[V(x)-\lambda] w-f^{\prime}(\varphi) w \\
L_{2} w=-\Delta w+[V(x)-\lambda] w-g\left(|\varphi|^{2}\right) w
\end{array}\right.
$$

Note here that $f^{\prime}(t)=g\left(|t|^{2}\right)+2 g^{\prime}\left(|t|^{2}\right) t^{2}$, so by assumption (H3) we have $L_{i} \in$ $\mathcal{L}\left(H^{1}, H^{-1}\right)$ for $i=1,2$. Similarly as noted in [19, p. 187], the orbital instability of the solitary wave solution $u_{\varphi}$ in (1.7) follows by the same argument as in the proof of [25, Theorem 6.2] once we have established the following.

Proposition 6.1. The operator

$$
\mathbf{M}:=J \mathrm{~d}^{2} \tilde{\Phi}_{\lambda}(\psi) \in \mathcal{L}\left(\mathcal{H}, \mathcal{H}^{*}\right)
$$

has a positive real eigenvalue, i.e., there exists $\rho>0$ and $\mathbf{w} \in \mathcal{H} \backslash\{0\}$ such that $\mathbf{M} \mathbf{w}=$ $\rho I \mathbf{w}$.

The remainder of this section is devoted to the proof of Proposition 6.1. We first note that

$$
L_{2} \varphi=0 \text { in } H^{-1} \text {, }
$$

since $\varphi$ is a critical point of $\left.\Phi\right|_{\Sigma_{\alpha}}$ with Lagrangian multiplier $\lambda$. Moreover, since $\lambda<$ $\inf \sigma_{\text {ess }}(-\Delta+V)$ by assumption, and since $g\left(|\varphi|^{2}\right)$ vanishes at infinity, Persson's Theorem [29, Theorem 14.11] implies that

$$
0<\inf \sigma_{\mathrm{ess}}(-\Delta+V-\lambda)=\inf \sigma_{\mathrm{ess}}\left(L_{2}\right)
$$

Since moreover $\varphi$ is a positive eigenfunction of $L_{2}$ corresponding to the eigenvalue 0 , it follows that $0=\inf \sigma\left(L_{2}\right)$ is a simple isolated eigenvalue. Consequently, putting

$$
\tilde{\Lambda}:=\left\{v \in H^{-1} \mid\langle v, \varphi\rangle_{*}=0\right\} \subset H^{-1}
$$


and

$$
\Lambda:=I^{-1}(\tilde{\Lambda})=\left\{v \in H^{1} \mid \int_{\mathbb{R}^{N}} v \varphi=0\right\} \subset H^{1},
$$

we see that the quadratic form $v \mapsto\left\langle L_{2} v, v\right\rangle_{*}$ is positive definite on $\Lambda$ and that $L_{2}$ defines an isomorphism $\Lambda \mapsto \tilde{\Lambda}$. From these properties, we deduce the following.

Lemma 6.2. We have $\left\langle I L_{2}^{-1} I v, v\right\rangle_{*}>0$ for all $v \in \Lambda \backslash\{0\}$.

Proof. Let $v \in \Lambda \backslash\{0\}$, then $I v \in \tilde{\Lambda}$ and by the remarks above there exists $\tilde{v} \in \Lambda \backslash\{0\}$ with $L_{2} \tilde{v}=I v$. Consequently, we have

$$
\left\langle I L_{2}^{-1} I v, v\right\rangle_{*}=\langle I \tilde{v}, v\rangle_{*}=\langle I v, \tilde{v}\rangle_{*}=\left\langle L_{2} \tilde{v}, \tilde{v}\right\rangle_{*}>0
$$

by the positive definiteness of the quadratic form $\tilde{v} \mapsto\left\langle L_{2} \tilde{v}, \tilde{v}\right\rangle_{*}$ on $\Lambda$.

The following lemma is the key step in the proof of Proposition 6.1. It resembles [19, Lemma 2.2], but we need to prove it by a different (more general) argument since our setting does not satisfy the assumptions in [19].

Lemma 6.3. We have

$$
\mu:=\inf _{v \in \Lambda \backslash\{0\}} \frac{\left\langle L_{1} v, v\right\rangle_{*}}{\left\langle I L_{2}^{-1} I v, v\right\rangle_{*}} \in(-\infty, 0) .
$$

Moreover, $\mu$ is attained at some $v \in \Lambda \backslash\{0\}$ satisfying the equation

$$
L_{1} v=\mu I L_{2}^{-1} I v+I \beta \varphi \quad \text { in } H^{-1} .
$$

for some $\beta \in \mathbb{R}$.

Proof. Since $\varphi$ has positive Morse index with respect to $\left.\Phi\right|_{\Sigma_{\alpha}}$, there exists $v \in \Lambda \backslash\{0\}$ with $\left\langle L_{1} v, v\right\rangle_{*}<0$, which implies that $\mu<0$. In the following, we consider the spectral decomposition

$$
\Lambda=V^{-} \oplus V^{+}
$$

with the properties that $\operatorname{dim} V^{-}<\infty$ and

$$
\left\langle L_{1} v, v\right\rangle_{*} \leq 0, \quad\left\langle L_{1} w, w\right\rangle_{*} \geq \delta\|w\|^{2}, \quad\left\langle L_{1} v, w\right\rangle_{*}=0 \quad \text { for } v \in V^{-}, w \in V^{+}
$$

with some $\delta>0$. The existence of such a decomposition follows from the fact that $\inf \sigma_{\text {ess }}\left(L_{1}\right)=\inf \sigma_{\text {ess }}(-\Delta+V-\lambda)>0$. For $v \in \Lambda$, we now write $v=v^{-}+v^{+}$with $v^{-} \in V^{-}, v^{+} \in V^{+}$. Let $\left(v_{n}\right)_{n} \subset \Lambda \backslash\{0\}$ be a minimizing sequence for the quotient

$$
v \mapsto q(v):=\frac{\left\langle L_{1} v, v\right\rangle_{*}}{\left\langle I L_{2}^{-1} I v, v\right\rangle_{*}} .
$$

Since $\mu=\inf _{v \in \Lambda \backslash\{0\}} q(v)<0$, we may assume that

$$
\left\langle L_{1} v_{n}, v_{n}\right\rangle_{*}=\left\langle L_{1} v_{n}^{-}, v_{n}^{-}\right\rangle_{*}+\left\langle L_{1} v_{n}^{+}, v_{n}^{+}\right\rangle_{*}<0 \quad \text { for all } n \in \mathbb{N} \text {. }
$$


Thus $v_{n}^{-} \neq 0$, and we may assume that $\left\|v_{n}^{-}\right\|=1$ for all $n \in \mathbb{N}$. Since $V^{-}$is finite dimensional, we may pass to a subsequence such that $v_{n}^{-} \rightarrow v_{-} \in V^{-}$with $\left\|v_{-}\right\|=1$. Then (6.3) and (6.4) imply that

$$
\delta \limsup _{n \rightarrow \infty}\left\|v_{n}^{+}\right\|^{2} \leq \limsup _{n \rightarrow \infty}\left\langle L_{1} v_{n}^{+}, v_{n}^{+}\right\rangle_{*} \leq-\lim _{n \rightarrow \infty}\left\langle L_{1} v_{n}^{-}, v_{n}^{-}\right\rangle_{*}=-\left\langle L_{1} v_{-}, v_{-}\right\rangle_{*}
$$

and thus $v_{n}^{+}$is bounded in $H^{1}$ as well. Hence $\left(v_{n}\right)_{n} \subset \Lambda$ is bounded in $H^{1}$, and we may thus pass to a subsequence such that

$$
\begin{aligned}
& v_{n}^{+} \rightarrow v_{+}, \quad v_{n} \rightarrow v:=v_{-}+v_{+} \in \Lambda \backslash\{0\}, \\
& \left\langle L_{1} v_{n}, v_{n}\right\rangle_{*} \rightarrow \kappa_{1} \leq 0 \quad \text { and } \quad\left\langle I L_{2}^{-1} I v_{n}, v_{n}\right\rangle_{*} \rightarrow \kappa_{2} \geq 0
\end{aligned}
$$

as $n \rightarrow \infty$. By weak lower semicontinuity, we then have

$$
\left\langle L_{1} v_{+}, v_{+}\right\rangle_{*} \leq \lim _{n \rightarrow \infty}\left\langle L_{1} v_{n}^{+}, v_{n}^{+}\right\rangle_{*}=\kappa_{1}-\left\langle L_{1} v_{-}, v_{-}\right\rangle_{*}
$$

and thus

$$
\left\langle L_{1} v, v\right\rangle_{*} \leq \kappa_{1} \leq 0
$$

Consequently, since also

$$
0<\left\langle I L_{2}^{-1} I v, v\right\rangle_{*} \leq \kappa_{2}
$$

by Lemma 6.2 and weak lower semicontinuity, we find that

$$
q(v)=\frac{\left\langle L_{1} v, v\right\rangle_{*}}{\left\langle I L_{2}^{-1} I v, v\right\rangle_{*}} \leq \frac{\left\langle L_{1} v, v\right\rangle_{*}}{\kappa_{2}} \leq \frac{\kappa_{1}}{\kappa_{2}}=\mu .
$$

Hence $v$ is a minimizer of $q$ in $\Lambda \backslash\{0\}$, and therefore $q(v)=\mu>-\infty$. Moreover, $v$ minimizes the functional

$$
\Lambda \rightarrow \mathbb{R}, \quad w \mapsto\left\langle L_{1} w-\mu I L_{2}^{-1} I w, w\right\rangle_{*}
$$

and therefore we have

$$
\left\langle L_{1} v-\mu I L_{2}^{-1} I v, w\right\rangle_{*}=0 \quad \text { for all } w \in \Lambda .
$$

This implies that there exists $\beta \in \mathbb{R}$ such that

$$
\left\langle L_{1} v-\mu I L_{2}^{-1} I v, w\right\rangle_{*}=\beta \int_{\mathbb{R}} \varphi w \quad \text { for all } w \in H^{1},
$$

i.e.,

$$
L_{1} v-\mu I L_{2}^{-1} I v=\beta I \varphi \quad \text { in } H^{-1}
$$

which gives (6.2).

Proof of Proposition 6.1 (completed). Let $\mu$ and $v$ be as in Lemma 6.3, let $\rho=\sqrt{-\mu}>0$, and consider

$$
\mathbf{w}=\left(\begin{array}{c}
v \\
-\rho L_{2}^{-1} I v+\rho^{-1} \beta \varphi
\end{array}\right) \in \mathcal{H} \backslash\{0\} .
$$

Then we have

$$
\mathbf{M} \mathbf{w}=\left(\begin{array}{cc}
0 & -L_{2} \\
L_{1} & 0
\end{array}\right) \mathbf{w}=\left(\begin{array}{c}
\rho I v \\
\mu I L_{2}^{-1} I v+I \beta \varphi
\end{array}\right)=\rho I \mathbf{w},
$$

so $\mathbf{w} \in \mathcal{H}$ is an eigenfunction of $\mathbf{M}$ corresponding to the eigenvalue $\rho>0$. 


\section{A. Proof of $(5.22)$}

In this section we compute the free Morse index of the rescaled single peak solutions $u_{\varepsilon}$ of (5.6) studied in Section 5. More precisely, we will prove the equality (5.22) for $\varepsilon>0$ small. We continue to use the notation from Section 5 . Recall that since $u_{\varepsilon}$ is a critical point of $\Phi_{\varepsilon}^{*}$ on $\sum_{\left|u_{\varepsilon}\right|_{2}^{2}}$ with Lagrange multiplier 0 , the free Morse index coincides with the Morse index of $u_{\varepsilon}$ as a critical point of $\Phi_{\varepsilon}^{*}$ in $H^{1}$. Recall moreover that $u_{\varepsilon}$ has a unique local maximum point $x_{\varepsilon}$, where $x_{\varepsilon} \rightarrow 0$ as $\varepsilon \rightarrow 0$ by [26, Proposition 5.2]. Put

$$
u_{0, \varepsilon}:=u_{0}\left(\cdot-x_{\varepsilon}\right)=\mathcal{T}_{x_{\varepsilon}} u_{0} \in H^{1} \quad \text { for } \varepsilon \in\left(0, \varepsilon_{0}\right) .
$$

We first need the following refined convergence estimate:

$$
\left\|u_{0, \varepsilon}-u_{\varepsilon}\right\|_{H^{2}}=O\left(\varepsilon^{2}\right) \quad \text { as } \varepsilon \rightarrow 0 .
$$

Suppose by contradiction that this is false, then along a sequence $\left(\varepsilon_{n}\right)_{n} \subset\left(0, \varepsilon_{0}\right)$ with $\varepsilon_{n} \rightarrow 0$ we have $d_{n}:=\left\|u_{0, \varepsilon_{n}}-u_{\varepsilon_{n}}\right\|_{H^{2}} \geq n \varepsilon_{n}^{2}$ for all $n \in \mathbb{N}$. Put $w_{n}:=\frac{u_{0, \varepsilon_{n}}-u_{\varepsilon_{n}}}{d_{n}}$; then $w_{n}$ is a weak solution of the equation

$$
-\Delta w_{n}+w_{n}=\frac{1}{d_{n}}\left(u_{0, \varepsilon_{n}}^{p-1}-u_{\varepsilon_{n}}^{p-1}+\left(V_{\varepsilon_{n}}-1\right) u_{\varepsilon_{n}}\right)=\tau_{n} w_{n}+\frac{V_{\varepsilon_{n}}-1}{d_{n}} u_{\varepsilon_{n}}
$$

with

$$
\tau_{n}(x)=(p-1) \int_{0}^{1}\left[(1-s) u_{0, \varepsilon_{n}}+s u_{\varepsilon_{n}}\right]^{p-2} \mathrm{~d} s .
$$

We pass to a subsequence such that $w_{n} \rightarrow w$ in $H^{2}$. Since $\tau_{n} \rightarrow(p-1) u_{0}^{p-2}$ as $n \rightarrow \infty$ uniformly in $\mathbb{R}^{N}$ by (5.10), and since

$$
\left|\frac{V_{\varepsilon_{n}}-1}{d_{n}} u_{\varepsilon_{n}}(x)\right| \leq \frac{c}{n}|x|^{2} \mathrm{e}^{-\alpha|x|} \quad \text { for } x \in \mathbb{R}^{N}, n \in \mathbb{N} \text { with constants } c, \alpha>0
$$

by (5.9) and (5.13), we may pass to the limit in (A.2) to see that $w$ is a (weak) solution of the equation

$$
-\Delta w+w-(p-1) u_{0}^{p-2} w=0 .
$$

Consequently, $w=\sum_{\ell=1}^{N} a_{\ell} \partial_{\ell} u_{0}$ with $\ell=1, \ldots, N$. However, since both $u_{0, \varepsilon_{n}}$ and $u_{\varepsilon_{n}}$ attain a maximum at $x_{\varepsilon_{n}}$, we infer from (A.2) and elliptic regularity that

$$
0=\lim _{n \rightarrow \infty} \partial_{j} w_{n}\left(x_{\varepsilon_{n}}\right)=\partial_{j} w(0)=\sum_{\ell=1}^{N} a_{\ell} \partial_{\ell j} u_{0}(0) \quad \text { for } j=1, \ldots, N .
$$

It is well known that 0 is the only maximum point of $u_{0}$, see, e.g., [40, Lemma 1(b)]. Considering that $u_{0}(x)=U_{0}(|x|)$, where $U_{0}$ is the solution with initial values $U_{0}(0)=$ $u_{0}(0)$ and $U_{0}^{\prime}(0)=0$ of the ordinary differential equation on $[0, \infty)$ corresponding to radial solutions of (5.3), and considering the uniqueness of solutions to that ODE, it is clear that 0 is a nondegenerate maximum point for $u_{0}$. Hence it follows that $a_{1}, \ldots, a_{N}=$ 0 and thus $w=0$. This implies that $w_{n} \rightarrow 0$ in $L_{\text {loc }}^{2}\left(\mathbb{R}^{N}\right)$, and thus

$$
-\Delta w_{n}+w_{n}=o(1) \quad \text { in } L^{2}\left(\mathbb{R}^{N}\right)
$$


by (A.2), (A.3), and since $\tau_{n}$ has exponential decay in $x$, uniformly in $n$. The boundedness of the inverse of $-\Delta+1$ on $L^{2}$ implies that $\left\|w_{n}\right\|_{H^{2}} \rightarrow 0$, contrary to the definition of $w_{n}$. Hence (A.1) follows.

We now consider the uniformly bounded families of linear operators

$$
A_{\varepsilon}:=\mathrm{D}^{2} \Phi_{\varepsilon}^{*}\left(u_{\varepsilon}\right) \in \mathcal{L}\left(H^{1}\right)
$$

and

$$
C_{\varepsilon}:=\mathcal{T}_{-x_{\varepsilon}} \circ A_{\varepsilon} \circ \mathcal{T}_{x_{\varepsilon}} \in \mathcal{L}\left(H^{1}\right), \quad \varepsilon \in\left(0, \varepsilon_{0}\right) .
$$

Here, as before, the symbol $\mathrm{D}^{2}$ denotes the derivative of the gradient with respect to the scalar product $\langle\cdot, \cdot\rangle_{H^{1}}$. The quadratic form associated with $A_{\varepsilon}$ is given by

$$
\left\langle A_{\varepsilon} v, w\right\rangle_{H^{1}}=\int_{\mathbb{R}^{N}}\left(\nabla v \cdot \nabla w+\left[V_{\varepsilon}-(p-1) u_{\varepsilon}^{p-2}\right] v w\right) \quad \text { for } v, w \in H^{1} .
$$

It is then clear that $A_{\varepsilon}$ and $C_{\varepsilon}$ share the same spectrum. We have

$$
\lim _{\varepsilon \rightarrow 0}\left\|C_{\varepsilon} v-B^{0} v\right\|_{H^{1}}=\lim _{\varepsilon \rightarrow 0}\left\|A_{\varepsilon} v-B^{0} v\right\|_{H^{1}}=0 \quad \text { for all } v \in H^{1},
$$

where, as before, $B^{0}=\mathrm{D}^{2} \Phi_{0}^{*}\left(u_{0}\right) \in \mathcal{L}\left(H^{1}\right)$, and the convergence is uniform on compact subsets of $H^{1}$. We claim that

$$
\left\|C_{\varepsilon} \partial_{i} u_{0}\right\|_{H^{1}}=O\left(\varepsilon^{2}\right) \quad \text { for } i=1, \ldots, N,
$$

and that

$$
\left\langle C_{\varepsilon} \partial_{i} u_{0}, \partial_{j} u_{0}\right\rangle_{H^{1}}=\frac{1}{2} \varepsilon^{2} \partial_{i j} V(0)\left|u_{0}\right|_{2}^{2}+o\left(\varepsilon^{2}\right) \quad \text { for } i, j=1, \ldots, N
$$

as $\varepsilon \rightarrow 0$. For this we recall that $\partial_{i} u_{\varepsilon}$ solves the equation

$$
-\Delta\left(\partial_{i} u_{\varepsilon}\right)+V_{\varepsilon} \partial_{j} u_{\varepsilon}-(p-1) u_{\varepsilon}^{p-2} \partial_{j} u_{\varepsilon}=-u_{\varepsilon} \partial_{j} V_{\varepsilon},
$$

and therefore (5.9) and (5.14) yield

$$
\begin{aligned}
A_{\varepsilon} \partial_{i} u_{\varepsilon}=(-\Delta+1)^{-1}\left(-\Delta\left(\partial_{i} u_{\varepsilon}\right)\right. & \left.+V_{\varepsilon} \partial_{i} u_{\varepsilon}-(p-1) u_{\varepsilon}^{p-2} \partial_{i} u_{\varepsilon}\right) \\
& =-(-\Delta+1)^{-1} u_{\varepsilon} \partial_{j} V_{\varepsilon}=O\left(\varepsilon^{2}\right) \quad \text { in } H^{1} .
\end{aligned}
$$

Combining this with (A.1), we find that

$$
\left\|C_{\varepsilon} \partial_{i} u_{0}\right\|_{H^{1}}=\left\|A_{\varepsilon} \partial_{i} u_{0, \varepsilon}\right\|_{H^{1}}=\left\|A_{\varepsilon} \partial_{i} u_{\varepsilon}\right\|_{H^{1}}+O\left(\varepsilon^{2}\right)=O\left(\varepsilon^{2}\right),
$$

as claimed in (A.6). To see (A.7), we note that

$$
\begin{aligned}
& \left\langle C_{\varepsilon} \partial_{i} u_{0}, \partial_{j} u_{0}\right\rangle_{H^{1}}=\left\langle A_{\varepsilon} \partial_{i} u_{0, \varepsilon}, \partial_{j} u_{0, \varepsilon}\right\rangle_{H^{1}} \\
& \quad=\left\langle A_{\varepsilon} \partial_{i} u_{\varepsilon}, \partial_{j} u_{\varepsilon}\right\rangle_{H^{1}}+\left\langle A_{\varepsilon} \partial_{i} u_{0, \varepsilon}, \partial_{j}\left(u_{0, \varepsilon}-u_{\varepsilon}\right)\right\rangle_{H^{1}}+\left\langle A_{\varepsilon} \partial_{j} u_{\varepsilon}, \partial_{i}\left(u_{0, \varepsilon}-u_{\varepsilon}\right)\right\rangle_{H^{1}}
\end{aligned}
$$


where, since $\partial_{i} u_{0, \varepsilon}$ satisfies $-\Delta \partial_{i} u_{0, \varepsilon}+\partial_{i} u_{0, \varepsilon}-(p-1) u_{0, \varepsilon}^{p-2} \partial_{i} u_{0, \varepsilon}=0$ in $\mathbb{R}^{N}$,

$$
\left\langle A_{\varepsilon} \partial_{i} u_{0, \varepsilon}, \partial_{j}\left(u_{0, \varepsilon}-u_{\varepsilon}\right)\right\rangle_{H^{1}}=\int_{\mathbb{R}^{N}}\left[V_{\varepsilon}-1+(p-1)\left(u_{0, \varepsilon}^{p-2}-u_{\varepsilon}^{p-2}\right)\right] \partial_{i} u_{0, \varepsilon} \partial_{j}\left(u_{0, \varepsilon}-u_{\varepsilon}\right)=o\left(\varepsilon^{2}\right)
$$

as $\varepsilon \rightarrow 0$. Here, in the last step, we used (A.1) together with the fact that

$$
\left\|\left[V_{\varepsilon}-1+(p-1)\left(u_{0, \varepsilon}^{p-2}-u_{\varepsilon}^{p-2}\right)\right] \partial_{i} u_{0, \varepsilon}\right\|_{L^{2}} \rightarrow 0 \quad \text { as } \varepsilon \rightarrow 0 .
$$

Moreover,

$$
\left|\left\langle A_{\varepsilon} \partial_{j} u_{\varepsilon}, \partial_{i}\left(u_{0, \varepsilon}-u_{\varepsilon}\right)\right\rangle_{H^{1}}\right| \leq\left\|A_{\varepsilon} \partial_{j} u_{\varepsilon}\right\|_{H^{1}}\left\|\partial_{i}\left(u_{0, \varepsilon}-u_{\varepsilon}\right)\right\|_{H^{1}} \leq O\left(\varepsilon^{4}\right)
$$

by (A.1) and (A.9). Inserting these estimates in (A.10) and using (A.8) once more, together with (5.9), (5.10), and (5.14) we find that

$$
\begin{aligned}
\left\langle C_{\varepsilon} \partial_{i} u_{0}, \partial_{j} u_{0}\right\rangle_{H^{1}} & =\left\langle A_{\varepsilon} \partial_{i} u_{\varepsilon}, \partial_{j} u_{\varepsilon}\right\rangle_{H^{1}}+o\left(\varepsilon^{2}\right)=-\int_{\mathbb{R}^{N}} u_{\varepsilon} \partial_{i} V_{\varepsilon} \partial_{j} u_{\varepsilon}+o\left(\varepsilon^{2}\right) \\
& =-\varepsilon^{2} \sum_{\ell=1}^{N} \partial_{i \ell} V(0) \int_{\mathbb{R}^{N}} x_{\ell} u_{0} \partial_{j} u_{0} \mathrm{~d} x+o\left(\varepsilon^{2}\right)=\frac{1}{2} \varepsilon^{2} \partial_{i j} V(0)\left|u_{0}\right|_{2}^{2}+o\left(\varepsilon^{2}\right) .
\end{aligned}
$$

In the last step we have integrated by parts again. This yields (A.7).

To conclude the proof of (5.22), we now put $X=\operatorname{span}\left(u_{0}\right), Y:=\operatorname{span}\left(\partial_{1} u_{0}, \ldots, \partial_{N} u_{0}\right)$, and we let $Z$ denote the $\langle\cdot, \cdot\rangle_{H^{1}}$-orthogonal complement of $X \oplus Y$ in $H^{1}$. We then have

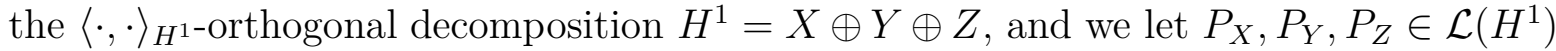
denote the corresponding orthogonal projections onto $X, Y$, and $Z$. It then follows from (A.6) that

$$
\left\|C_{\varepsilon} P_{Y}\right\|_{\mathcal{L}\left(H^{1}\right)}=O\left(\varepsilon^{2}\right) \quad \text { as } \varepsilon \rightarrow 0 .
$$

Moreover, by the remarks before Lemma 5.2, there exists $0<\delta<1$ such that

$$
\left\langle B^{0} u_{0}, u_{0}\right\rangle_{H^{1}} \leq-\delta \quad \text { and } \quad\left\langle B^{0} w, w\right\rangle_{H^{1}} \geq \delta\|w\|_{H^{1}}^{2} \quad \text { for all } w \in Z .
$$

It then follows from (A.5) that

$$
\left\langle C_{\varepsilon} u_{0}, u_{0}\right\rangle_{H^{1}}<-\frac{\delta}{2} \quad \text { for } \varepsilon>0 \text { sufficiently small. }
$$

We also claim that

$$
\inf _{w \in Z,\|w\|_{H^{1}}=1}\left\langle C_{\varepsilon} w, w\right\rangle_{H^{1}}>\delta_{+}:=\frac{1}{2} \min \left\{\delta, \inf _{\mathbb{R}^{N}} V\right\} \quad \text { for } \varepsilon>0 \text { sufficiently small. }
$$

Indeed, suppose by contradiction there exist $\varepsilon_{n} \in\left(0, \varepsilon_{0}\right)$ and $w_{n} \in Z$ with $\left\|w_{n}\right\|_{H^{1}}=1$ for $n \in \mathbb{N}$ such that $\varepsilon_{n} \rightarrow 0$ as $n \rightarrow \infty$ and

$$
\left\langle C_{\varepsilon_{n}} w_{n}, w_{n}\right\rangle_{H^{1}} \leq \delta_{+} \quad \text { as } n \rightarrow \infty
$$


Passing to a subsequence, we may then assume that $w_{n} \rightarrow w$ in $H^{1}$ with $w \in Z$. We put $\tilde{w}_{n}:=\mathcal{T}_{x_{\varepsilon_{n}}} w_{n}=w_{n}\left(\cdot-x_{\varepsilon_{n}}\right)$ for $n \in \mathbb{N}$, then also $\tilde{w}_{n} \rightarrow w$, and we may pass to a subsequence such that $\tilde{w}_{n} \rightarrow w$ in $L_{\text {loc }}^{2}\left(\mathbb{R}^{N}\right)$ and $\tilde{w}_{n} \rightarrow w$ pointwise a.e. on $\mathbb{R}^{N}$. By (5.9) and (5.10) this implies that

$$
\int_{\mathbb{R}^{N}} u_{\varepsilon_{n}}^{p-2} \tilde{w}_{n}^{2} \rightarrow \int_{\mathbb{R}^{N}} u_{0}^{p-2} w^{2} \quad \text { as } n \rightarrow \infty .
$$

We also have that

$\int_{\mathbb{R}^{N}}\left(\left|\nabla\left(\tilde{w}_{n}-w\right)\right|^{2}+V_{\varepsilon_{n}}\left(\tilde{w}_{n}-w\right)^{2}\right)=o(1)+\int_{\mathbb{R}^{N}}\left(\left|\nabla \tilde{w}_{n}\right|^{2}-|\nabla w|^{2}+V_{\varepsilon_{n}}\left[\tilde{w}_{n}^{2}-w^{2}-2\left(\tilde{w}_{n}-w\right) w\right]\right)$,

where, since $\left|\tilde{w}_{n}-w\right| \rightarrow 0$ in $L^{2}\left(\mathbb{R}^{N}\right)$,

$$
\left|\int_{\mathbb{R}^{N}} V_{\varepsilon_{n}}\left(\tilde{w}_{n}-w\right) w\right| \leq\|V\|_{L^{\infty}\left(\mathbb{R}^{N}\right)} \int_{\mathbb{R}^{N}}\left|\tilde{w}_{n}-w \| w\right| \rightarrow 0 \quad \text { as } n \rightarrow \infty .
$$

Moreover,

$$
\int_{\mathbb{R}^{N}} V_{\varepsilon_{n}} w^{2} \rightarrow \int_{\mathbb{R}^{N}} w^{2} \quad \text { as } n \rightarrow \infty
$$

by (5.13) and Lebesgue's theorem. Consequently,

$$
\begin{aligned}
\int_{\mathbb{R}^{N}} & \left(\left|\nabla \tilde{w}_{n}\right|^{2}+V_{\varepsilon_{n}} \tilde{w}_{n}^{2}\right) \\
& =\int_{\mathbb{R}^{N}}\left(|\nabla w|^{2}+w^{2}\right)+\int_{\mathbb{R}^{N}}\left(\left|\nabla\left(\tilde{w}_{n}-w\right)\right|^{2}+V_{\varepsilon_{n}}\left(\tilde{w}_{n}-w\right)^{2}\right)+o(1) \\
& \geq\|w\|_{H^{1}}^{2}+\min \left\{1, \inf _{\mathbb{R}^{N}} V\right\}\left\|\tilde{w}_{n}-w\right\|_{H^{1}}^{2}+o(1) \geq\|w\|_{H^{1}}^{2}+2 \delta_{+}\left\|\tilde{w}_{n}-w\right\|_{H^{1}}^{2}+o(1),
\end{aligned}
$$

and together with (A.4), (A.12) and (A.16) this implies that

$$
\begin{aligned}
& \left\langle C_{\varepsilon_{n}} w_{n}, w_{n}\right\rangle_{H^{1}}=\left\langle A_{\varepsilon_{n}} \tilde{w}_{n}, \tilde{w}_{n}\right\rangle_{H^{1}} \geq\left\langle B^{0} w, w\right\rangle_{H^{1}}+2 \delta_{+}\left\|\tilde{w}_{n}-w\right\|_{H^{1}}^{2}+o(1) \\
& \geq 2 \delta_{+}\|w\|_{H^{1}}^{2}+2 \delta_{+}\left\|\tilde{w}_{n}-w\right\|_{H^{1}}^{2}+o(1)=2 \delta_{+}\left\|w_{n}\right\|_{H^{1}}^{2}+o(1)=2 \delta_{+}+o(1) .
\end{aligned}
$$

This contradicts (A.15), and hence (A.14) follows.

In the following, we let $M \in \mathbb{R}^{N \times N}$ denote the Hessian of the potential $V$ at 0 which is nondegenerate by assumption. Then there exists a basis of eigenvectors $b^{1}, \ldots, b^{N} \in \mathbb{R}^{N}$ of $M$ corresponding to the eigenvalues $\mu_{1} \leq \cdots \leq \mu_{N}$, where

$$
\mu_{i}<0 \quad \text { for } i \leq m_{V} \quad \text { and } \quad \mu_{i}>0 \quad \text { for } i>m_{V} .
$$

We then let $w^{1}, \ldots, w^{N} \in \operatorname{span}\left(\partial_{1} u_{0}, \ldots, \partial_{N} u_{0}\right)$ be defined by

$$
w^{i}:=\sum_{j=1}^{N} b_{j}^{i} \partial_{j} u_{0} \quad \text { for } i=1, \ldots, N \text {, }
$$

and we define the subspaces $\tilde{Y}_{ \pm} \subset Y$ by

$$
\tilde{Y}_{-}:=\operatorname{span}\left(w^{1}, \ldots, w^{m}\right) \quad \text { and } \quad \tilde{Y}_{+}:=\operatorname{span}\left(w^{m+1}, \ldots, w^{N}\right) .
$$


By (A.7) and construction, there exists $\tilde{\delta}>0$ such that for $\varepsilon>0$ sufficiently small we have

(A.17)

$$
\left\langle C_{\varepsilon} w, w\right\rangle_{H^{1}} \leq-\tilde{\delta} \varepsilon^{2}\|w\|_{H^{1}}^{2} \text { for } w \in \tilde{Y}_{-} \quad \text { and } \quad\left\langle C_{\varepsilon} w, w\right\rangle_{H^{1}} \geq \tilde{\delta} \varepsilon^{2}\|w\|_{H^{1}}^{2} \text { for } w \in \tilde{Y}_{+} .
$$

We now consider the spaces

$$
\tilde{X}:=\operatorname{span}\left(u_{0}\right) \oplus \tilde{Y}_{-} \quad \text { and } \quad \tilde{Z}:=Z \oplus \tilde{Y}_{+} .
$$

Then (5.22) follows once we have shown that

$$
\sup _{w \in \tilde{X},\|w\|_{H^{1}}=1}\left\langle C_{\varepsilon} w, w\right\rangle_{H^{1}}<0
$$

and

$$
\inf _{w \in \tilde{Z},\|w\|_{H^{1}}=1}\left\langle C_{\varepsilon} w, w\right\rangle_{H^{1}}>0
$$

for $\varepsilon>0$ sufficiently small. We only show (A.19), the proof of (A.18) is very similar but simpler. Suppose by contradiction that (A.19) does not hold true for $\varepsilon>0$ sufficiently small. Then there exist $\varepsilon_{n} \in\left(0, \varepsilon_{0}\right)$ and $w_{n} \in \tilde{Z}$ with $\left\|w_{n}\right\|_{H^{1}}=1$ for $n \in \mathbb{N}$ such that $\varepsilon_{n} \rightarrow 0$ as $n \rightarrow \infty$ and

$$
\left\langle C_{\varepsilon_{n}} w_{n}, w_{n}\right\rangle_{H^{1}} \leq 0 \quad \text { as } n \rightarrow \infty
$$

With $w_{n}^{1}:=P_{Z} w_{n} \in Z$ and $w_{n}^{2}:=P_{Y} w_{n} \in \tilde{Y}^{+}$we have, by (A.11), (A.14) and (A.17),

$$
\begin{aligned}
\left\langle C_{\varepsilon_{n}} w_{n}, w_{n}\right\rangle_{H^{1}} & =\left\langle C_{\varepsilon_{n}} w_{n}^{1}, w_{n}^{1}\right\rangle_{H^{1}}+\left\langle C_{\varepsilon_{n}} w_{n}^{2}, w_{n}^{2}\right\rangle_{H^{1}}+2\left\langle C_{\varepsilon_{n}} w_{n}^{2}, w_{n}^{1}\right\rangle_{H^{1}} \\
& \geq \delta_{+}\left\|w_{n}^{1}\right\|_{H^{1}}^{2}+\tilde{\delta}\left\|w_{n}^{2}\right\|_{H^{1}}^{2} \varepsilon_{n}^{2}+O\left(\left\|w_{n}^{1}\right\|_{H^{1}} \varepsilon_{n}^{2}\right) .
\end{aligned}
$$

Passing to a subsequence, we may assume that either $\left\|w_{n}^{1}\right\|_{H^{1}} \rightarrow 0$ and $\left\|w_{n}^{2}\right\|_{H^{1}} \rightarrow 1$ as $n \rightarrow \infty$, or that $\left\|w_{n}^{1}\right\|_{H^{1}} \geq c$ for some constant $c>0$ and all $n \in \mathbb{N}$. In the first case, we deduce that

$$
\left\langle C_{\varepsilon_{n}} w_{n}, w_{n}\right\rangle_{H^{1}} \geq \tilde{\delta} \varepsilon_{n}^{2}+o\left(\varepsilon_{n}^{2}\right)
$$

and in the second case we obtain that

$$
\left\langle C_{\varepsilon_{n}} w_{n}, w_{n}\right\rangle_{H^{1}} \geq \delta_{+} c^{2}+o(1)
$$

as $n \rightarrow \infty$. In both cases we arrive at a contradiction to (A.20), and thus (A.19) is proved. As remarked before, (A.18) is obtained similarly by using (A.13) and the first inequality in (A.17). The proof of (5.22) is thus finished. 


\section{References}

[1] N. Ackermann, A nonlinear superposition principle and multibump solutions of periodic Schrödinger equations, J. Funct. Anal. 234 (2006), no. 2, 277-320. MR MR2216902

[2] N. Ackermann, Uniform continuity and Brézis-Lieb type splitting for superposition operators in Sobolev space, Adv. Nonlinear Anal. (2016).

[3] N. Ackermann and T. Weth, Multibump solutions of nonlinear periodic Schrödinger equations in a degenerate setting, Commun. Contemp. Math. 7 (2005), no. 3, 269298. MR MR2151860

[4] A. Aftalion and B. Helffer, On mathematical models for Bose-Einstein condensates in optical lattices, Rev. Math. Phys. 21 (2009), no. 2, 229-278. MR 2502397 (2010m:82010)

[5] S. Alama and Y.Y. Li, On "multibump" bound states for certain semilinear elliptic equations, Indiana Univ. Math. J. 41 (1992), no. 4, 983-1026. MR 94d:35044

[6] G. Arioli, A. Szulkin, and W. Zou, Multibump solutions and critical groups, Trans. Amer. Math. Soc. 361 (2009), no. 6, 3159-3187. MR 2485422 (2010h:37139)

[7] B.B. Baizakov, B.A. Malomed, and M. Salerno, Multidimensional solitons in periodic potentials, EPL (Europhysics Letters) 63 (2003), no. 5, 642.

[8] T. Bartsch and S. de Valeriola, Normalized solutions of nonlinear Schrödinger equations, Arch. Math. (Basel) 100 (2013), no. 1, 75-83. MR 3009665

[9] T. Bartsch and N. Soave, A natural constraint approach to normalized solutions of nonlinear Schrödinger equations and systems, J. Funct. Anal. 272 (2017), no. 12, 4998-5037. MR 3639521

[10] J.V. Baxley and J.C. Moorhouse, Lagrange multiplier problems in economics, Amer. Math. Monthly 91 (1984), no. 7, 404-412. MR 759215

[11] J. Bellazzini, N. Boussaïd, L. Jeanjean, and N. Visciglia, Existence and Stability of Standing Waves for Supercritical NLS with a Partial Confinement, Comm. Math. Phys. 353 (2017), no. 1, 229-251. MR 3638314

[12] J. Bellazzini and L. Jeanjean, On dipolar quantum gases in the unstable regime, SIAM J. Math. Anal. 43 (2016), no. 3, 2028-2058.

[13] T. Cazenave and P.L. Lions, Orbital stability of standing waves for some nonlinear Schrödinger equations, Comm. Math. Phys. 85 (1982), no. 4, 549-561. MR 677997 (84i:81015) 
[14] V. Coti Zelati and P.H. Rabinowitz, Homoclinic type solutions for a semilinear elliptic PDE on $\mathbf{R}^{n}$, Comm. Pure Appl. Math. 45 (1992), no. 10, 1217-1269. MR 93k:35087

[15] F. Dalfovo, S. Giorgini, L.P. Pitaevskii, and S. Stringari, Theory of Bose-Einstein condensation in trapped gases, Rev. Mod. Phys. 71 (1999), 463-512.

[16] K. Deimling, Nonlinear functional analysis, Springer-Verlag, Berlin, 1985. MR MR787404 (86j:47001)

[17] C.H. Edwards, Jr., Advanced calculus of several variables, Dover Publications, Inc., New York, 1994, Corrected reprint of the 1973 original. MR 1319337

[18] N.K. Efremidis and D.N. Christodoulides, Lattice solitons in Bose-Einstein condensates, Phys. Rev. A 67 (2003), 063608.

[19] M.J. Esteban and W.A. Strauss, Nonlinear bound states outside an insulated sphere, Comm. Partial Differential Equations 19 (1994), no. 1-2, 177-197. MR 1257002

[20] J.W. Fleischer, M. Segev, N.K. Efremidis, and D.N. Christodoulides, Observation of two-dimensional discrete solitons in optically induced nonlinear photonic lattices, Nature 422 (2003), 147-150.

[21] R. Fukuizumi, Stability and instability of standing waves for the nonlinear Schrödinger equation with harmonic potential, Discrete Contin. Dynam. Systems 7 (2001), no. 3, 525-544. MR 1815766

[22] R. Fukuizumi and M. Ohta, Instability of standing waves for nonlinear Schrödinger equations with potentials, Differential Integral Equations 16 (2003), no. 1, 111-128. MR 1948875

[23] L. Greenberg, J.H. Maddocks, and K.A. Hoffman, The bordered operator and the index of a constrained critical point, Math. Nachr. 219 (2000), 109-124. MR 1791915

[24] M. Grillakis, J. Shatah, and W. Strauss, Stability theory of solitary waves in the presence of symmetry. I, J. Funct. Anal. 74 (1987), no. 1, 160-197. MR 901236 (88g:35169)

[25] _ Stability theory of solitary waves in the presence of symmetry. II, J. Funct. Anal. 94 (1990), no. 2, 308-348. MR 1081647

[26] M. Grossi, On the number of single-peak solutions of the nonlinear Schrödinger equation, Ann. Inst. H. Poincaré Anal. Non Linéaire 19 (2002), no. 3, 261-280. MR 1956951

[27] C. Hassell and E. Rees, The index of a constrained critical point, Amer. Math. Monthly 100 (1993), no. 8, 772-778. MR 1237226 
[28] K.M. Hilligsøe, M.K. Oberthaler, and K.P. Marzlin, Stability of gap solitons in a Bose-Einstein condensate, Phys. Rev. A 66 (2002), 063605.

[29] P.D. Hislop and I.M. Sigal, Introduction to spectral theory, Applied Mathematical Sciences, vol. 113, Springer-Verlag, New York, 1996, With applications to Schrödinger operators. MR 1361167

[30] J.B. Hughes, Second order sufficient conditions for optimizing with equality constraints, Math. Comput. Modelling 15 (1991), no. 12, 29-36. MR 1145891

[31] I. Ianni and S. Le Coz, Orbital stability of standing waves of a semiclassical nonlinear Schrödinger-Poisson equation, Adv. Differential Equations 14 (2009), no. 7-8, 717-748. MR 2527691 (2010f:35390)

[32] L. Jeanjean, Existence of solutions with prescribed norm for semilinear elliptic equations, Nonlinear Anal. 28 (1997), no. 10, 1633-1659. MR 1430506

[33] L. Jeanjean and M. Squassina, An approach to minimization under a constraint: the added mass technique, Calc. Var. Partial Differential Equations 41 (2011), no. 3-4, 511-534. MR 2796242

[34] W. Kryszewski and A. Szulkin, Infinite-dimensional homology and multibump solutions, J. Fixed Point Theory Appl. 5 (2009), no. 1, 1-35. MR MR2491945

[35] T.C. Lin and J. Wei, Orbital stability of bound states of semiclassical nonlinear Schrödinger equations with critical nonlinearity, SIAM J. Math. Anal. 40 (2008), no. 1, 365-381. MR 2403325

[36] P.L. Lions, The concentration-compactness principle in the calculus of variations. The locally compact case. II, Ann. Inst. H. Poincaré Anal. Non Linéaire 1 (1984), no. 4, 223-283. MR 778974 (87e:49035b)

[37] P.J.Y. Louis, E.A. Ostrovskaya, C.M. Savage, and Y.S. Kivshar, Bose-Einstein condensates in optical lattices: Band-gap structure and solitons, Phys. Rev. A 67 (2003), 013602.

[38] J.H. Maddocks, Restricted quadratic forms and their application to bifurcation and stability in constrained variational principles, SIAM J. Math. Anal. 16 (1985), no. 1, 47-68. MR 772868

[39] _ Errata: "Restricted quadratic forms and their application to bifurcation and stability in constrained variational principles" [SIAM J. Math. Anal. 16 (1985), no. 1, 47-68; MR0772868 (86f:49111)], SIAM J. Math. Anal. 19 (1988), no. 5, 12561257. MR 957686

[40] K. McLeod, Uniqueness of positive radial solutions of $\Delta u+f(u)=0$ in $\mathbf{R}^{n}$. II, Trans. Amer. Math. Soc. 339 (1993), no. 2, 495-505. MR 94b:35105 
[41] O. Morsch and M. Oberthaler, Dynamics of Bose-Einstein condensates in optical lattices, Rev. Mod. Phys. 78 (2006), 179-215.

[42] W.M. Ni and I. Takagi, Locating the peaks of least-energy solutions to a semilinear Neumann problem, Duke Math. J. 70 (1993), no. 2, 247-281. MR 94 h:35072

[43] E.A. Ostrovskaya and Y.S. Kivshar, Matter-wave gap solitons in atomic band-gap structures, Phys. Rev. Lett. 90 (2003), 160407.

[44] R.S. Palais, Morse theory on Hilbert manifolds, Topology 2 (1963), 299-340. MR 0158410

[45] P.H. Rabinowitz, A variational approach to multibump solutions of differential equations, Hamiltonian dynamics and celestial mechanics (Seattle, WA, 1995), Contemp. Math., vol. 198, Amer. Math. Soc., Providence, RI, 1996, pp. 31-43. MR 97j:58051

[46] _ A multibump construction in a degenerate setting, Calc. Var. Partial Differential Equations 5 (1997), no. 2, 159-182. MR 98b:58034

[47] P. Shutler, Constrained critical points, Amer. Math. Monthly 102 (1995), no. 1, 49-52. MR 1321457

[48] G.S. Spradlin, Multibump solutions to a class of semilinear elliptic partial differential equations, Ph.D. thesis, University of Wisconsin-Madison, 1995.

[49] D. Spring, On the second derivative test for constrained local extrema, Amer. Math. Monthly 92 (1985), no. 9, 631-643. MR 1465784

[50] C.A. Stuart, Lectures on the orbital stability of standing waves and application to the nonlinear Schrödinger equation, Milan J. Math. 76 (2008), 329-399. MR 2465996 (2009k:37165) 